\title{
The association between vitamin D concentration and pain: a systematic review and meta-analysis
}

\author{
Zhenqiang Wu, Zarintaj Malihi, Alistair W Stewart, Carlene MM Lawes and \\ Robert Scragg* \\ School of Population Health, University of Auckland, Private Bag 92019, Auckland, 1142, New Zealand
}

Submitted 19 April 2017: Final revision received 31 January 2018: Accepted 12 February 2018: First published online 21 March 2018

\begin{abstract}
Objective: Pain-related conditions, such as chronic widespread pain and fibromyalgia, are major burdens for individuals and the health system. Evidence from previous research on the association between circulating 25-hydroxyvitamin D (25(OH)D) concentrations and pain is conflicting. Thus, we aimed to determine if there is an association between mean 25(OH)D concentration (primary aim), or proportion of hypovitaminosis D (secondary aim), and pain conditions in observational studies. Design: Published observational research on $25(\mathrm{OH}) \mathrm{D}$ concentration and pain-related conditions was systematically searched for in electronic sources (MEDLINE, EMBASE and Cochrane Central Register of Controlled Trials) and a random-effects meta-analysis was conducted on included studies.

Results: Eighty-one observational studies with a total of 50834 participants were identified. Compared with controls, mean 25(OH)D concentration was significantly lower in patients with arthritis (mean difference (MD): $-12.34 \mathrm{nmol} / \mathrm{l}$; $P<0.001$ ), muscle pain (MD: $-8.97 \mathrm{nmol} / 1 ; P=0.003)$ and chronic widespread pain (MD: $-7.77 \mathrm{nmol} / 1 ; P<0 \cdot 001$ ), but not in patients with headache or migraine (MD: $-2.53 \mathrm{nmol} / 1 ; P=0.06)$. The odds of vitamin D deficiency was increased for arthritis, muscle pain and chronic widespread pain, but not for headache or migraine, compared with controls. Sensitivity analyses revealed similar results. Conclusions: A significantly lower $25(\mathrm{OH}) \mathrm{D}$ concentration was observed in patients with arthritis, muscle pain and chronic widespread pain, compared with those without. These results suggest that low 25(OH)D concentrations may be associated with pain conditions.
\end{abstract}

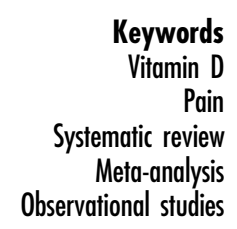

Pain is defined by the International Association for the Study of Pain as 'an unpleasant sensory and emotional experience associated with actual or potential tissue damage, or described in terms of such damage, ${ }^{,(1)}$. Pain, and conditions with pain as a prominent symptom, including chronic widespread pain, musculoskeletal pain (e.g. lower-back pain), arthritis and headache, are the most common reason for primary care and medical consultations ${ }^{(2-4)}$. The prevalence of chronic pain ranges from 8 to $60 \%$ and over, depending on the population studied $^{(5)}$. Recent reviews of painful conditions have reported that the prevalence of fibromyalgia varies from 2 to $8 \%$ in the general population ${ }^{(6)}$; while the global prevalence of lowerback pain is $9.4 \%{ }^{(7)}$, of rheumatoid arthritis is $0.24 \%^{(8)}$ and of current migraine is more than $10 \%$ in adults ${ }^{(4)}$. Painful conditions can seriously influence quality of life, lead to work disability, and result in major economic burdens for both individuals and the health system ${ }^{(2,4,5,8-12)}$.
Vitamin D comprises a group of fat-soluble secosteroids ${ }^{(13)}$ and the receptor of vitamin $\mathrm{D}$ has been identified in muscle tissue ${ }^{(14)}$. Although the optimal level of serum 25-hydroxyvitamin D (25(OH)D) is a topic of ongoing research, vitamin $\mathrm{D}$ deficiency is typically defined as $25(\mathrm{OH}) \mathrm{D}<50 \mathrm{nmol} / \mathrm{l}^{(15)}$. Vitamin D deficiency is very common in both developed ${ }^{(16)}$ and developing countries ${ }^{(17)}$. There is increasing evidence from observational studies that vitamin D deficiency is associated with a wide range of acute and chronic diseases ${ }^{(18)}$, including diseases with pain as a prominent symptom ${ }^{(19)}$. Previous research has provided inconsistent findings on the association between vitamin D status and pain. A meta-analysis of seven observational studies with 2420 statin-treated patients found that $25(\mathrm{OH}) \mathrm{D}$ levels were lower in those with myalgia than in those without ${ }^{(20)}$. However, it used a fixed-effects model, not justified by the high heterogeneity of the results $\left(I^{2}=94 \%\right)$, which when 
repeated with a random-effects model was no longer significant (mean difference (MD): $-3.52 ; 95 \% \mathrm{CI}-8.55,1.51 \mathrm{ng} / \mathrm{ml}$; $P=0 \cdot 17$ ). Another meta-analysis of twelve observational studies with 1854 participants reported inconsistent results, with significantly increased odds of vitamin $\mathrm{D}$ deficiency associated with chronic widespread pain, but no difference in mean 25(OH)D levels between people with and without chronic widespread pain ${ }^{(21)}$. Moreover, these previous meta-analyses have not reported the association between 25(OH)D concentration and other pain-related conditions, such as arthritis and headache. In addition, recent reviews of randomized controlled trials have reported inconsistent conclusions about whether vitamin D supplementation improves chronic pain, with two being qualitative reviews ${ }^{(22,23)}$ and only one using quantitative methods ${ }^{(24)}$.

Given the limited evidence and inconsistent conclusions from previous reviews and meta-analyses, which for observational studies only searched up to September $2014^{(20,21)}$, we conducted an updated meta-analysis of all observational studies reporting data on 25(OH)D levels and pain, including studies of non-statin users and participants with different pain conditions, to determine if there is an association between these two variables.

\section{Methods}

\section{Search strategy}

Two trained researchers (Z.W., Z.M.) independently searched MEDLINE, EMBASE and Cochrane Central Register of Controlled Trials (up to April 2017) using the following key words: Vitamin D, Vitamin D2, Vitamin D3, Cholecalciferol, Ergocalciferol, 25-hydroxyvitamin D, Pain, Myalgia, Myopathy, Myalgic, Headache, Migraine, Arthritis and Sciatica, for original publications pertinent to vitamin D levels and pain (search strategy listed in the online supplementary material, Supplement 1). In addition, we manually searched the reference lists of eligible articles and previous reviews for additional studies.

\footnotetext{
Aims

Two aims were predefined in the meta-analysis. The primary aim was the difference in mean circulating $25(\mathrm{OH}) \mathrm{D}$ concentration $(\mathrm{nmol} / \mathrm{l})$ between participants with and without pain-related conditions. The secondary aim was the difference in proportions of hypovitaminosis $\mathrm{D}$ in the participants with and without painful conditions. For the latter, we used the original definition of hypovitaminosis $\mathrm{D}$ from each paper (eight studies with a threshold of $75 \mathrm{nmol} / 1$; thirty-four studies with $50 \mathrm{nmol} / 1$; two studies with $25 \mathrm{nmol} / 1$; six studies with other definitions, which were $20 \mathrm{nmol} / 1,30 \mathrm{nmol} / \mathrm{l}$, $37.5 \mathrm{nmol} / 1,80 \mathrm{nmol} / \mathrm{l}$ and $100 \mathrm{nmol} / \mathrm{l})$.
}

\section{Eligibility criteria}

We included observational studies in the current metaanalysis if the study: (i) was a cohort, case-control or cross-sectional study; (ii) enrolled adult participants ( $\geq 18$ years old); (iii) described specific information on pain, such as a pain definition or category; and (iv) reported the 25(OH)D level and/or the proportion of hypovitaminosis $\mathrm{D}$ in participants with and without pain. There was no language or ethnicity restriction. In addition, the studies which selected controls with pain conditions were excluded.

\section{Data extraction}

Reviewers (Z.W., Z.M.) independently identified the included articles by screening title, abstract and full text ( $\kappa$ coefficient $=0.73$ ), and the main data were extracted based on a standardized data collection form developed for the study. Any inconsistencies were resolved by consensus and discussion.

\section{Quality assessment of individual studies}

The quality of each included study was assessed using the Newcastle-Ottawa scale ${ }^{(25)}$. Specifically, there were five items for cross-sectional studies, and eight items for cohort and case-control studies. We used the same score to categorize the quality of studies as reported previously ${ }^{(26)}$ : 5 as very good, 4 as good, 3 as satisfactory and $0-2$ as unsatisfactory in cross-sectional studies; similarly, $7-8$ as very good, 5-6 as good, 4 as satisfactory and $0-3$ as unsatisfactory in case-control or cohort studies.

\section{Synthesis and analysis}

Mean and SD of serum 25(OH)D levels, and number of participants with and without pain, were collected for the continuous exposure measurement. All 25(OH)D levels were transformed to $\mathrm{nmol} / \mathrm{l}$ in the meta-analysis. Digitizer software (GetData Graph Digitizer version 2.26; www. getdata-graph-digitizer.com/) was used to extract the data from graphs, and Wan et al.'s ${ }^{(27)}$ methods were used to estimate the mean and sD by reported median and range, or median and interquartile range. Sample size and the proportion with hypovitaminosis D were collected for the dichotomous exposure measurement.

Weighted MD and 95\% CI were calculated for continuous exposure, and OR and $95 \%$ CI were calculated for dichotomous exposure, to allow the combining of different study designs in the synthesis analysis. Heterogeneity was measured using Cochran's $Q$ test and the $I^{2}$ statistic $\left(I^{2}>50 \%\right.$ denotes large or extreme heterogeneity). Random-effects models were used in the meta-analysis ${ }^{(28)}$. Predefined analyses were performed to detect the relationship of $25(\mathrm{OH}) \mathrm{D}$ with pain by different painful conditions (arthritis, muscle pain, chronic widespread pain, and headache or migraine), type of study design, statin user and different cut-off points of vitamin D deficiency $(25 \mathrm{nmol} / 1,50 \mathrm{nmol} / 1$ and $75 \mathrm{nmol} / \mathrm{l})$. Interactions were tested between different subgroups using a standard $\operatorname{method}^{(29)}$. In addition, we also conducted metaregression to examine other sources of heterogeneity (e.g. year, sample size, mean age, female proportion, type 
of study). Sensitivity analyses were also conducted by individually excluding each study in turn, and by collectively excluding low-quality studies or those that used other definitions of vitamin D deficiency (as listed above). We generated funnel plots for visual assessment of publication bias, as well as performed the Egger test ${ }^{(30)}$. All tests were two-tailed and $P \leq 0.05$ was considered statistically significant. We conducted the meta-analysis using the Stata statistical software package version 13.1 and Review Manager software (Revman version 5.2).

\section{Results}

\section{Included studies}

A total of 2340 unique articles were identified by searching the three electronic databases and by applying snowballing techniques. After reviewing titles and abstracts, 2155 publications were excluded. The full texts of the remaining 185 studies were assessed for their eligibility and a further 107 were excluded because they did not meet the eligibility criteria (see Supplement 1). The remaining seventy-eight publications, which reported eighty-one observational studies with data on vitamin D concentration and pain, were included in the current meta-analysis (Fig. 1).

The eighty-one observational studies involved nineteen cross-sectional studies ${ }^{(31-48)}$, fifty-six case-control studies $^{(49-102)}$ and six cohort studies ${ }^{(103-108)}$. Together, these studies included 50834 participants, 21723 of whom were reported as pain subjects, with a median mean age of 49.4 (median SD 10.3) years and median female proportion of 80.5\% (range: 0-100\%); and 29111 participants who were community- or hospital-based controls without pain-related conditions, with a median mean age of $50 \cdot 0$ (median SD 10.3) years and median female proportion of $78.4 \%$ (range: $0-100 \%$ ). The pain conditions or symptoms reported in these studies included arthritis, muscle pain, chronic widespread pain, and headache or migraine. Characteristics of included studies are shown in Table 1.

\section{Quality assessment in included studies}

According to the Newcastle-Ottawa scale, sixty-two of the eighty-one observational studies were very good or
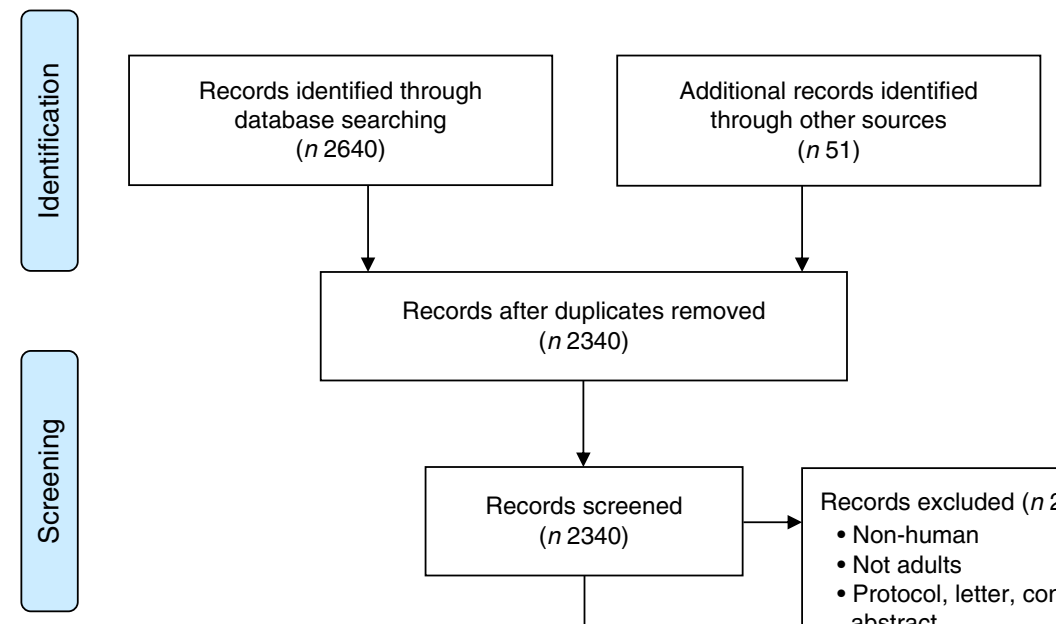

Records after duplicates removed (n 2340)
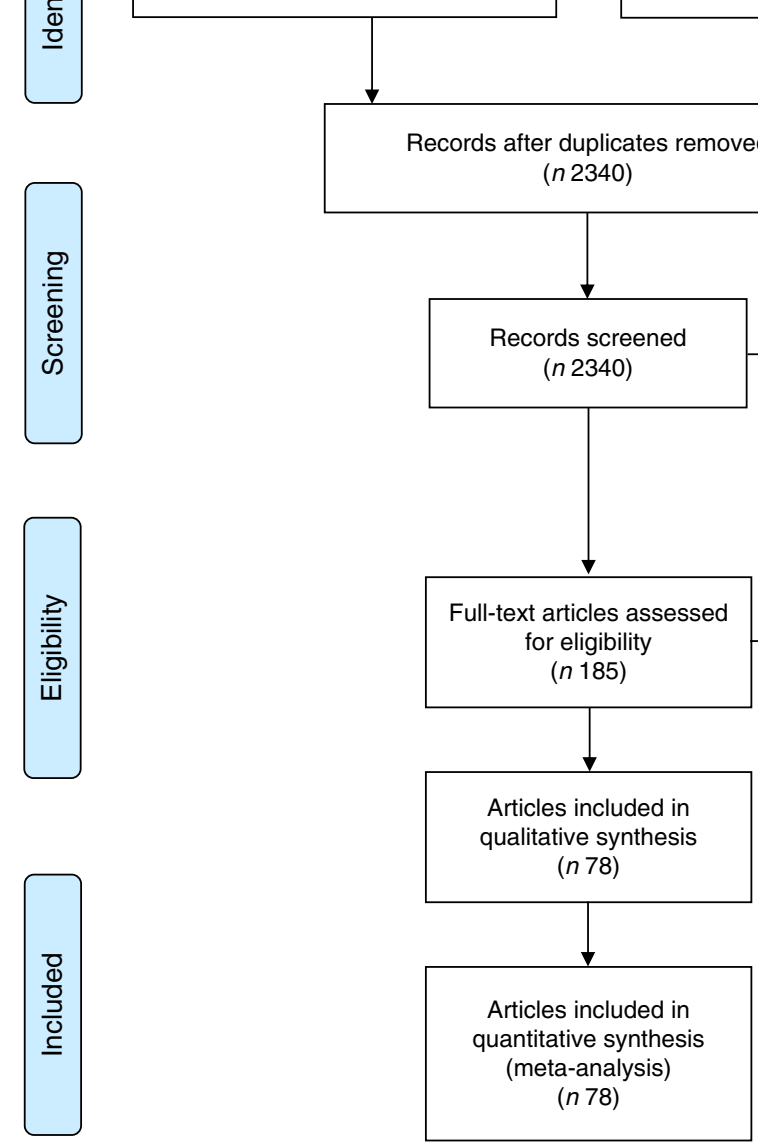

Records excluded ( $n$ 2155):

- Non-human

- Not adults

- Protocol, letter, conference abstract

- Not relevant to vitamin D

- Not relevant to pain

Full-text articles excluded, with reasons ( $n$ 107):

- Not relevant $(n 7)$

- Review articles ( $n$ 14)

- Abstract only (n 18)

- No useful data (n 47)

- Only included patients with

pain ( $n 7)$

- Controls with pain ( $n 3)$

- Age less than 18 years ( $n 9)$

- Data from same study (n 2)

Fig. 1 (colour online) PRISMA (Preferred Reporting Items for Systematic Reviews and Meta-Analyses) diagram of study selection 
Table 1 Characteristics of studies included in the current meta-analysis on the association between vitamin D concentration and pain

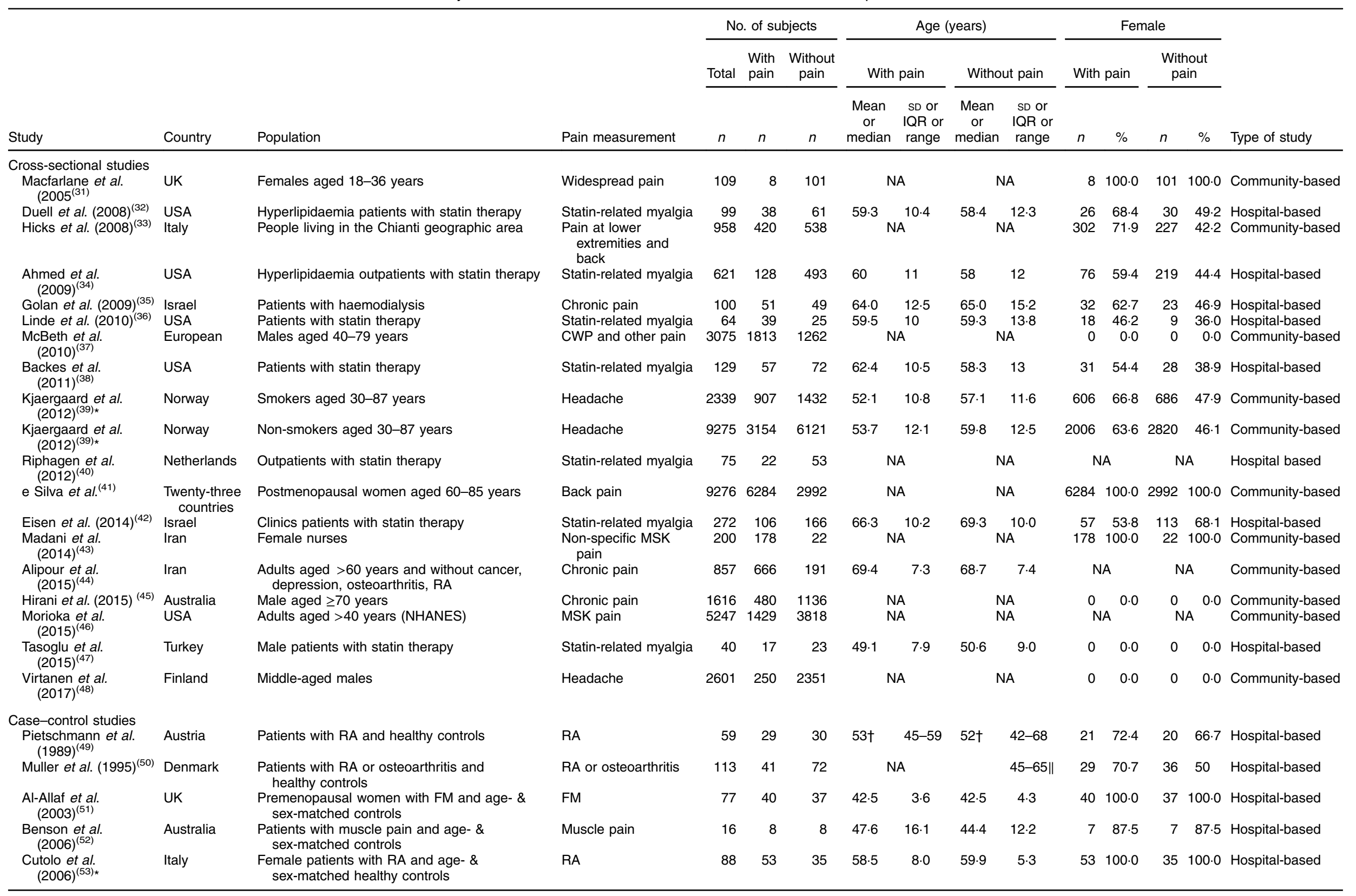




\begin{tabular}{|c|c|c|c|c|c|c|c|c|c|c|c|c|c|c|c|}
\hline \multirow[b]{3}{*}{ Study } & \multirow[b]{3}{*}{ Country } & \multirow[b]{3}{*}{ Population } & \multirow[b]{3}{*}{ Pain measurement } & \multicolumn{3}{|c|}{ No. of subjects } & \multicolumn{4}{|c|}{ Age (years) } & \multicolumn{4}{|c|}{ Female } & \multirow[b]{3}{*}{ Type of study } \\
\hline & & & & \multirow{2}{*}{$\begin{array}{c}\text { Total } \\
n\end{array}$} & \multirow{2}{*}{$\begin{array}{c}\text { With } \\
\text { pain }\end{array}$} & \multirow{2}{*}{$\begin{array}{c}\begin{array}{c}\text { Without } \\
\text { pain }\end{array} \\
n\end{array}$} & \multicolumn{2}{|c|}{ With pain } & \multicolumn{2}{|c|}{ Without pain } & \multicolumn{2}{|c|}{ With pain } & \multicolumn{2}{|c|}{$\begin{array}{l}\text { Without } \\
\text { pain }\end{array}$} & \\
\hline & & & & & & & $\begin{array}{l}\text { Mean } \\
\text { or } \\
\text { median }\end{array}$ & $\begin{array}{l}\text { SD or } \\
\text { IQR or } \\
\text { range }\end{array}$ & $\begin{array}{l}\text { Mean } \\
\text { or } \\
\text { median }\end{array}$ & $\begin{array}{l}\text { SD or } \\
\text { IQR or } \\
\text { range }\end{array}$ & $n$ & $\%$ & $n$ & $\%$ & \\
\hline $\begin{array}{l}\text { Cutolo et al. } \\
(2006)^{(53) *}\end{array}$ & Estonia & $\begin{array}{l}\text { Female patients with RA and age- \& sex-matched } \\
\text { healthy controls }\end{array}$ & RA & 94 & 64 & 30 & $56 \cdot 3$ & $18 \cdot 4$ & $51 \cdot 1$ & $20 \cdot 8$ & 64 & $100 \cdot 0$ & 30 & $100 \cdot 0$ & Hospital-based \\
\hline Lotfi et al. $(2007)^{(54)}$ & Egypt & $\begin{array}{l}\text { Female patients with LBP and age- \& } \\
\text { sex-matched health controls }\end{array}$ & Chronic LBP & 80 & 60 & 20 & $32 \cdot 8$ & $7 \cdot 1$ & $33 \cdot 6$ & 8.6 & 60 & $100 \cdot 0$ & 20 & $100 \cdot 0$ & Hospital-based \\
\hline $\begin{array}{l}\text { Tandeter et al. } \\
(2009)^{(55)}\end{array}$ & Israel & $\begin{array}{l}\text { Premenopausal women with FM and age- \& } \\
\text { sex-matched women }\end{array}$ & FM & 150 & 68 & 82 & $43 \cdot 8$ & $7 \cdot 6$ & $40 \cdot 4$ & 9.9 & 68 & $100 \cdot 0$ & 82 & $100 \cdot 0$ & Hospital-based \\
\hline $\begin{array}{l}\text { de Rezende Pena } \\
\text { et al. }(2010)^{(56)}\end{array}$ & Brazil & $\begin{array}{l}\text { Female patients with FM and age- \& sex-matched } \\
\text { controls }\end{array}$ & $\mathrm{FM}$ & 179 & 87 & 92 & $44 \cdot 9$ & 8.6 & $32 \cdot 0$ & $10 \cdot 5$ & 87 & $100 \cdot 0$ & 92 & $100 \cdot 0$ & Hospital-based \\
\hline $\begin{array}{l}\text { Heidari et al. } \\
(2010)^{(57)}\end{array}$ & Iran & $\begin{array}{l}\text { Outpatients with non-specific skeletal pain } \\
\text { and controls }\end{array}$ & $\begin{array}{l}\text { Non-specific skeletal } \\
\text { pain }\end{array}$ & 478 & 276 & 202 & $44 \cdot 3$ & 15 & $46 \cdot 4$ & $14 \cdot 2$ & \multicolumn{2}{|c|}{ NA } & \multicolumn{2}{|c|}{ NA } & Hospital-based \\
\hline $\begin{array}{l}\text { Turhanoglu et al. } \\
(2011)^{(58)}\end{array}$ & Turkey & Patients with RA and healthy controls & RA & 105 & 65 & 40 & $46 \cdot 3$ & 11.9 & 44.8 & $10 \cdot 6$ & \multicolumn{2}{|c|}{ NA } & \multicolumn{2}{|c|}{ NA } & Hospital-based \\
\hline Attar $(2012)^{(59)}$ & Saudi Arabia & $\begin{array}{l}\text { Patients with RA and age- \& sex-matched healthy } \\
\text { controls }\end{array}$ & RA & 200 & 100 & 100 & 47 & 13 & 47 & 15 & 90 & $90 \cdot 0$ & 89 & $89 \cdot 0$ & Hospital-based \\
\hline $\begin{array}{l}\text { Baykal et al. } \\
(2012)^{(60)}\end{array}$ & Turkey & $\begin{array}{l}\text { Patients with RA and age- \& sex-matched healthy } \\
\text { controls }\end{array}$ & RA & 100 & 55 & 45 & $45 \ddagger$ & $28-68$ & & NA & 40 & $72 \cdot 7$ & 33 & $73 \cdot 3$ & Hospital-based \\
\hline Dong et al. $(2012)^{(61)}$ & China & Female patients with RA and healthy controls & RA & 130 & 72 & 58 & 59.5 & $5 \cdot 3$ & $58 \cdot 8$ & $5 \cdot 1$ & & $100 \cdot 0$ & & $100 \cdot 0$ & Hospital-based \\
\hline $\begin{array}{l}\text { Heidari et al. } \\
(2012)^{(62)}\end{array}$ & Iran & $\begin{array}{l}\text { Patients with inflammatory arthritis (RA and UIA) } \\
\text { and controls }\end{array}$ & $\begin{array}{l}\text { Inflammatory arthritis } \\
\text { (RA and UIA) }\end{array}$ & 386 & 147 & 239 & $47 \cdot 1$ & 14.9 & 49.4 & 14.5 & & & & VA & Hospital-based \\
\hline $\begin{array}{l}\text { Kostoglou- } \\
\text { Athanassiou et al. } \\
(2012)^{(63)}\end{array}$ & Greece & $\begin{array}{l}\text { Patients with RA and age- \& sex-matched } \\
\text { controls }\end{array}$ & RA & 88 & 44 & 44 & \multicolumn{2}{|c|}{ NA } & \multicolumn{2}{|c|}{ NA } & \multicolumn{2}{|c|}{ NA } & \multicolumn{2}{|c|}{ NA } & Hospital-based \\
\hline $\begin{array}{l}\text { Al-Jarallah et al. } \\
\qquad(2013)^{(64)}\end{array}$ & Kuwait & $\begin{array}{l}\text { Rheumatology/rehabilitation clinics patients with } \\
\text { MSK pain and age- \& sex-matched healthy } \\
\text { controls }\end{array}$ & MSK pain & 206 & 124 & 82 & $41 \cdot 7$ & 13.9 & 43.7 & $7 \cdot 4$ & 118 & $95 \cdot 2$ & 79 & $96 \cdot 3$ & Hospital-based \\
\hline Atwa et al. $(2013)^{(65)}$ & Saudi Arabia & $\begin{array}{l}\text { Patients with RA and age-matched healthy } \\
\text { controls }\end{array}$ & RA & 95 & 55 & 40 & $45 \cdot 6$ & $12 \cdot 4$ & $45 \cdot 0$ & $8 \cdot 0$ & 43 & $78 \cdot 2$ & 20 & $50 \cdot 0$ & Hospital-based \\
\hline Azali et al. $(2013)^{(66)}$ & Sweden & $\begin{array}{l}\text { Patients with IIM and sex- } \& \text { month of blood } \\
\text { sample-matched healthy controls }\end{array}$ & IIM & 439 & 149 & 290 & $56 \S$ & $18-72$ & $41 \S$ & $18-70$ & 97 & $65 \cdot 1$ & 192 & $66 \cdot 2$ & Hospital-based \\
\hline Olama et al. $(2013)^{(67)}$ & Egypt & $\begin{array}{l}\text { Female patients with FM and age- \& } \\
\text { sex-matched healthy controls }\end{array}$ & $\mathrm{FM}$ & 100 & 50 & 50 & $32 \cdot 3$ & 9.4 & 33.1 & 9.7 & 50 & $100 \cdot 0$ & 50 & $100 \cdot 0$ & Hospital-based \\
\hline $\begin{array}{l}\text { Orgaz-Molina et al. } \\
(2013)^{(68)}\end{array}$ & Spain & $\begin{array}{l}\text { Psoriasis patients with arthritis and age- \& } \\
\text { sex-matched psoriasis without arthritis }\end{array}$ & Arthritis & 122 & 61 & 61 & 44.9 & $10 \cdot 9$ & 45.6 & 11.7 & 28 & $45 \cdot 9$ & 28 & $45 \cdot 9$ & Hospital-based \\
\hline Rkain et al. $(2013)^{(69)}$ & Morocco & $\begin{array}{l}\text { Postmenopausal women with chronic LBP and } \\
\text { age-, sex- \& BMI-matched healthy controls }\end{array}$ & Chronic LBP & 149 & 105 & 44 & 56.5 & $5 \cdot 6$ & $56 \cdot 8$ & $7 \cdot 4$ & 105 & $100 \cdot 0$ & 44 & $100 \cdot 0$ & Hospital-based \\
\hline $\begin{array}{l}\text { Yazmalar et al. } \\
(2013)^{(70)}\end{array}$ & Turkey & $\begin{array}{l}\text { Patients with RA or osteoarthritis and healthy } \\
\text { controls }\end{array}$ & RA or osteoarthritis & 215 & 145 & 70 & $47 \cdot 0$ & 9.1 & 41.4 & $4 \cdot 2$ & 99 & $68 \cdot 3$ & 26 & $37 \cdot 1$ & Hospital-based \\
\hline $\begin{array}{l}\text { Baykara et al. } \\
(2014)^{(71)}\end{array}$ & Turkey & $\begin{array}{l}\text { Patients with chronic non-specific LBP and } \\
\text { heathy controls }\end{array}$ & $\begin{array}{l}\text { Chronic non-specific } \\
\text { LBP }\end{array}$ & 90 & 60 & 30 & $30 \cdot 6$ & $7 \cdot 8$ & $31 \cdot 0$ & $6 \cdot 7$ & 37 & $61 \cdot 7$ & 19 & $63 \cdot 3$ & Hospital-based \\
\hline $\begin{array}{l}\text { Celikbilek et al. } \\
(2014)^{(72)}\end{array}$ & Turkey & $\begin{array}{l}\text { Patients with migraine and age- \& sex-matched } \\
\text { healthy people }\end{array}$ & Migraine & 101 & 52 & 49 & 35.9 & 9.1 & $34 \cdot 2$ & $10 \cdot 2$ & 48 & $92 \cdot 3$ & 42 & $85 \cdot 7$ & Hospital-based \\
\hline Chen et al. $(2014)^{(73)}$ & China & $\begin{array}{l}\text { Patients with RA and age- \& sex-matched healthy } \\
\text { controls }\end{array}$ & RA & 220 & 110 & 110 & 59.5 & $11 \cdot 4$ & $56 \cdot 9$ & $10 \cdot 5$ & 75 & $68 \cdot 2$ & 71 & 64.5 & Hospital-based \\
\hline Cote et al. $(2014)^{(74)}$ & USA & $\begin{array}{l}\text { Patients with RA and age- \& sex-matched non- } \\
\text { RA controls }\end{array}$ & RA & 1611 & 270 & 1341 & $\mathrm{~N}$ & JA & & NA & 225 & 83.3 & 1125 & 83.9 & Community-based \\
\hline
\end{tabular}




\begin{tabular}{|c|c|c|c|c|c|c|c|c|c|c|c|c|c|c|c|}
\hline \multirow[b]{3}{*}{ Study } & \multirow[b]{3}{*}{ Country } & \multirow[b]{3}{*}{ Population } & \multirow[b]{3}{*}{ Pain measurement } & \multicolumn{3}{|c|}{ No. of subjects } & \multicolumn{4}{|c|}{ Age (years) } & \multicolumn{4}{|c|}{ Female } & \multirow[b]{3}{*}{ Type of study } \\
\hline & & & & \multirow{2}{*}{ 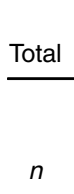 } & \multirow{2}{*}{$\begin{array}{c}\text { With } \\
\text { pain } \\
\\
n\end{array}$} & \multirow{2}{*}{$\begin{array}{c}\begin{array}{c}\text { Without } \\
\text { pain }\end{array} \\
\qquad \\
n\end{array}$} & \multicolumn{2}{|c|}{ With pain } & \multicolumn{2}{|c|}{ Without pain } & \multicolumn{2}{|c|}{ With pain } & \multicolumn{2}{|c|}{$\begin{array}{l}\text { Without } \\
\text { pain }\end{array}$} & \\
\hline & & & & & & & $\begin{array}{l}\text { Mean } \\
\text { or } \\
\text { median }\end{array}$ & $\begin{array}{l}\text { SD or } \\
\text { IQR or } \\
\text { range }\end{array}$ & $\begin{array}{l}\text { Mean } \\
\text { or } \\
\text { median }\end{array}$ & $\begin{array}{l}\text { SD or } \\
\text { IQR or } \\
\text { range }\end{array}$ & $n$ & $\%$ & $n$ & $\%$ & \\
\hline $\begin{array}{l}\text { Heidari et al. } \\
(2014)^{(75)}\end{array}$ & Iran & Patients with unexplained arthralgia and controls & Arthralgia & 453 & 167 & 286 & 38 & $13 \cdot 3$ & $42 \cdot 6$ & $14 \cdot 4$ & 135 & 80.8 & 221 & $77 \cdot 3$ & Hospital-based \\
\hline Hiraki et al. (2014) ${ }^{(76) \star}$ & USA & $\begin{array}{l}\text { Female patients with RA and age-, sex-, date } \\
\text { of blood draw- } \& \text { hormonal factors-matched } \\
\text { controls from NHS }\end{array}$ & RA & 477 & 120 & 357 & $56 \cdot 0$ & $7 \cdot 1$ & 56.0 & $7 \cdot 1$ & 120 & $100 \cdot 0$ & 357 & $100 \cdot 0$ & Community-based \\
\hline Hiraki et al. $(2014)^{(76) \star}$ & USA & $\begin{array}{l}\text { Female patients with RA and age-, sex-, date } \\
\text { of blood draw- } \& \text { hormonal factors-matched } \\
\text { controls from NHSII }\end{array}$ & RA & 179 & 46 & 133 & 44.4 & 4.4 & 44.5 & $5 \cdot 3$ & 46 & $100 \cdot 0$ & 133 & $100 \cdot 0$ & Community-based \\
\hline Hong et al. $(2014)^{(77)}$ & China & $\begin{array}{l}\text { Patients with RA and age- \& sex-matched healthy } \\
\text { controls }\end{array}$ & RA & 210 & 130 & 80 & 54 & 14 & 54 & 13 & 95 & $73 \cdot 1$ & 57 & 71.3 & Hospital-based \\
\hline $\begin{array}{l}\text { Mateos et al. } \\
(2014)^{(78)}\end{array}$ & Spain & $\begin{array}{l}\text { Female patients with FM and age-, sex- \& } \\
\text { enrolled year-matched healthy controls }\end{array}$ & $\mathrm{FM}$ & 410 & 205 & 205 & 51.5 & $9 \cdot 6$ & $51 \cdot 3$ & 9.9 & 205 & $100 \cdot 0$ & 205 & $100 \cdot 0$ & Hospital-based \\
\hline $\begin{array}{l}\text { Sezgin Ozcan et al. } \\
(2014)^{(79)}\end{array}$ & Turkey & $\begin{array}{l}\text { Female patients with FM and age- \& sex-matched } \\
\text { healthy controls }\end{array}$ & $\mathrm{FM}$ & 90 & 60 & 30 & 41.9 & $9 \cdot 8$ & $38 \cdot 8$ & $12 \cdot 7$ & 60 & $100 \cdot 0$ & 30 & $100 \cdot 0$ & Hospital-based \\
\hline $\begin{array}{l}\text { Sharma et al. } \\
(2014)^{(80)}\end{array}$ & India & $\begin{array}{l}\text { Patients with RA and age- \& sex-matched healthy } \\
\text { controls }\end{array}$ & RA & 160 & 80 & 80 & $40 \cdot 98$ & $12 \cdot 53$ & $42 \cdot 64$ & $12 \cdot 67$ & \multicolumn{2}{|c|}{ NA } & \multicolumn{2}{|c|}{ NA } & Hospital-based \\
\hline $\begin{array}{l}\text { Zandifar et al. } \\
(2014)^{(81)}\end{array}$ & Iran & $\begin{array}{l}\text { Patients with migraine and age- } \& \text { sex-matched } \\
\text { healthy controls }\end{array}$ & Migraine & 215 & 105 & 110 & $33 \cdot 6$ & $9 \cdot 9$ & 32.5 & 9.5 & 80 & $76 \cdot 2$ & 89 & 80.9 & Hospital-based \\
\hline $\begin{array}{l}\text { Brance et al. } \\
\quad(2015)^{(82)}\end{array}$ & Argentina & $\begin{array}{l}\text { Female patients with RA and age-, sex- \& } \\
\text { BMI-matched healthy controls }\end{array}$ & RA & 75 & 34 & 41 & $52 \cdot 2$ & $11 \cdot 1$ & $54 \cdot 8$ & $10 \cdot 9$ & 34 & $100 \cdot 0$ & 41 & $100 \cdot 0$ & Hospital-based \\
\hline Cen et al. (2015) & China & Patients with RA and normal controls & RA & 166 & 116 & 50 & $50 \cdot 1$ & $10 \cdot 9$ & $48 \cdot 1$ & $10 \cdot 3$ & 93 & $80 \cdot 2$ & 40 & $80 \cdot 0$ & Hospital-based \\
\hline Gullo et al. $(2015)^{(84)}$ & Italy & $\begin{array}{l}\text { Patients with RA and age- \& sex-matched } \\
\text { controls }\end{array}$ & RA & 68 & 27 & 41 & 47.5 & 12.5 & $46 \cdot 4$ & $4 \cdot 1$ & 19 & $70 \cdot 4$ & 28 & 68.3 & Hospital-based \\
\hline Lodh et al. (2015) ${ }^{(85)}$ & India & Patients with chronic LBP and controls & Chronic LBP & 400 & 200 & 200 & $46 \cdot 2$ & $15 \cdot 7$ & \multicolumn{2}{|c|}{ NA } & 146 & 73 & \multicolumn{2}{|c|}{ NA } & Hospital-based \\
\hline$\underset{(2015)^{(86)}}{\text { Matsumot }}$ et al. & Japan & $\begin{array}{l}\text { Outpatients with RA and age- \& sex-matched } \\
\text { controls }\end{array}$ & RA & 367 & 181 & 186 & $61 \dagger$ & $51-69$ & $60 \dagger$ & $51-66$ & 151 & 83.4 & 155 & 83.3 & Hospital-based \\
\hline Park et al. $(2015)^{(87)}$ & South Korea & $\begin{array}{l}\text { Patients with EIA and age- \& sex-matched } \\
\text { healthy controls }\end{array}$ & EIA & 202 & 101 & 101 & 56.5 & $12 \cdot 2$ & $56 \cdot 6$ & $12 \cdot 1$ & 86 & $85 \cdot 1$ & 86 & $85 \cdot 1$ & Hospital-based \\
\hline Petho et al. $(2015)^{(88)}$ & Hungary & $\begin{array}{l}\text { Male patients with psoriasis arthritis and } \\
\text { age- \& sex-matched healthy controls }\end{array}$ & Psoriatic arthritis & 106 & 53 & 53 & $54 \cdot 7 \ddagger$ & $31-84$ & $54 \cdot 7 \ddagger$ & $31-84$ & 0 & 0.0 & 0 & 0.0 & Hospital-based \\
\hline Yagiz et al. (2015) ${ }^{(89)}$ & Turkey & Patients with RA and healthy controls & RA & 154 & 92 & 62 & 49.6 & 13.9 & 43.9 & 8.0 & 83 & $90 \cdot 2$ & 35 & 56.5 & Hospital-based \\
\hline Askari et al. $(2016)^{(90)}$ & Iran & $\begin{array}{l}\text { Patients with keen osteoarthritis and sex- } \\
\text { matched healthy controls }\end{array}$ & Osteoarthritis & 393 & 131 & 262 & 52.0 & 8.0 & 55.0 & 9.0 & 107 & 81.7 & 214 & 81.7 & Hospital-based \\
\hline $\begin{array}{l}\text { Elbassiony et al. } \\
\qquad(2016)^{(91)}\end{array}$ & Egypt & $\begin{array}{l}\text { Consecutive patients with RA and age- \& } \\
\text { sex-matched healthy controls }\end{array}$ & RA & 300 & 150 & 150 & 44.2 & 11.6 & $46 \cdot 4$ & $12 \cdot 9$ & 97 & $64 \cdot 7$ & 97 & 64.7 & Hospital-based \\
\hline Gamal et al. (2016) & Egypt & $\begin{array}{l}\text { Patients with RA and age- \& sex-matched healthy } \\
\text { controls }\end{array}$ & RA & 80 & 55 & 25 & $42 \cdot 2$ & $10 \cdot 6$ & $41 \cdot 2$ & $15 \cdot 8$ & 47 & 85.5 & 20 & $80 \cdot 0$ & Hospital-based \\
\hline Gheita et al. $(2016)^{(93)}$ & Egypt & $\begin{array}{l}\text { Patients with RA and age- \& sex-matched healthy } \\
\text { controls }\end{array}$ & RA & 125 & 63 & 62 & $41 \cdot 6$ & $9 \cdot 7$ & 39.7 & $9 \cdot 8$ & 49 & $77 \cdot 8$ & 49 & 79.0 & Hospital-based \\
\hline $\begin{array}{l}\text { Kasapoğlu Aksoy } \\
\text { et al. }(2017)^{(94)}\end{array}$ & Turkey & $\begin{array}{l}\text { Patients with FM and age- \& sex-matched healthy } \\
\text { controls }\end{array}$ & $\mathrm{FM}$ & 100 & 53 & 47 & $46 \cdot 4$ & $9 \cdot 8$ & $44 \cdot 4$ & $7 \cdot 6$ & 51 & $96 \cdot 2$ & 41 & 87.2 & Hospital-based \\
\hline Liao et al. $(2016)^{(95)}$ & China & Female patients with RA and healthy controls & RA & 114 & 82 & 32 & $54 \cdot 0$ & $14 \cdot 0$ & $53 \cdot 0$ & $14 \cdot 0$ & 82 & $100 \cdot 0$ & 32 & $100 \cdot 0$ & Hospital-based \\
\hline Maafi et al. $(2016)^{(96)}$ & Iran & Female patients with FM and healthy controls & FM & 142 & 74 & 68 & 38.0 & $9 \cdot 8$ & 32.6 & $10 \cdot 1$ & 74 & $100 \cdot 0$ & 68 & $100 \cdot 0$ & Hospital-based \\
\hline Okyay et al. $(2016)^{(97)}$ & Turkey & Female patients with FM and healthy controls & FM & 159 & 79 & 80 & 37.0 & 9.0 & $35 \cdot 8$ & $10 \cdot 7$ & 79 & $100 \cdot 0$ & 80 & $100 \cdot 0$ & Hospital-based \\
\hline $\begin{array}{l}\text { Thorneby et al. } \\
(2016)^{(98)}\end{array}$ & Sweden & $\begin{array}{l}\text { Patients with chronic LBP and age- \& sex- } \\
\text { matched controls }\end{array}$ & Chronic LBP & 88 & 44 & 44 & 55.0 & $16 \cdot 0$ & $55 \cdot 0$ & $15 \cdot 0$ & 26 & 59.1 & 26 & $59 \cdot 1$ & Community-based \\
\hline
\end{tabular}




\begin{tabular}{|c|c|c|c|c|c|c|c|c|c|c|c|c|c|c|c|}
\hline \multirow[b]{3}{*}{ Study } & \multirow[b]{3}{*}{ Country } & \multirow[b]{3}{*}{ Population } & \multirow[b]{3}{*}{ Pain measurement } & \multicolumn{3}{|c|}{ No. of subjects } & \multicolumn{4}{|c|}{ Age (years) } & \multicolumn{4}{|c|}{ Female } & \multirow[b]{3}{*}{ Type of study } \\
\hline & & & & \multirow{2}{*}{ 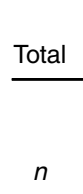 } & \multirow{2}{*}{$\begin{array}{c}\text { With } \\
\text { pain } \\
\\
n\end{array}$} & \multirow{2}{*}{$\begin{array}{c}\begin{array}{c}\text { Without } \\
\text { pain }\end{array} \\
\end{array}$} & \multicolumn{2}{|c|}{ With pain } & \multicolumn{2}{|c|}{ Without pain } & \multicolumn{2}{|c|}{ With pain } & \multicolumn{2}{|c|}{$\begin{array}{l}\text { Without } \\
\text { pain }\end{array}$} & \\
\hline & & & & & & & $\begin{array}{l}\text { Mean } \\
\text { or } \\
\text { median }\end{array}$ & $\begin{array}{l}\text { SD or } \\
\text { IQR or } \\
\text { range }\end{array}$ & $\begin{array}{l}\text { Mean } \\
\text { or } \\
\text { median }\end{array}$ & $\begin{array}{l}\text { SD or } \\
\text { IQR or } \\
\text { range }\end{array}$ & $n$ & $\%$ & $n$ & $\%$ & \\
\hline Wang et al. $(2016)^{(99)}$ & China & $\begin{array}{l}\text { Patients with RA and age- \& sex-matched healthy } \\
\text { controls }\end{array}$ & RA & 214 & 154 & 60 & 53.5 & $12 \cdot 4$ & $51 \cdot 4$ & $10 \cdot 3$ & 88 & $57 \cdot 1$ & 35 & $58 \cdot 3$ & Hospital-based \\
\hline $\begin{array}{l}\text { Yildirim et al. } \\
(2016)^{(100)}\end{array}$ & Turkey & $\begin{array}{l}\text { Patients with FM and age- \& sex-matched healthy } \\
\text { controls }\end{array}$ & $\mathrm{FM}$ & 198 & 99 & 99 & $49 \cdot 4$ & $9 \cdot 2$ & $50 \cdot 8$ & $8 \cdot 8$ & 80 & $80 \cdot 8$ & 77 & $77 \cdot 8$ & Hospital-based \\
\hline $\begin{array}{l}\text { Brennan-Speranzaa } \\
\text { et al. }(2017)^{(101)}\end{array}$ & Australia & $\begin{array}{l}\text { Patients with keen osteoarthritis and age- } \\
\text { matched controls }\end{array}$ & Osteoarthritis & 29 & 19 & 10 & $66 \cdot 1$ & $5 \cdot 2$ & $64 \cdot 7$ & $7 \cdot 6$ & 10 & 52.6 & 7 & $70 \cdot 0$ & Hospital-based \\
\hline $\begin{array}{l}\text { Wong et al. } \\
\qquad(2017)^{(102)}\end{array}$ & Malaysia & $\begin{array}{l}\text { Female patients with RA and age-matched } \\
\text { healthy controls }\end{array}$ & RA & 106 & 77 & 29 & $54 \cdot 1$ & 6.9 & $52 \cdot 6$ & $5 \cdot 4$ & 77 & $100 \cdot 0$ & 29 & $100 \cdot 0$ & Hospital-based \\
\hline \multicolumn{16}{|l|}{ Cohort studies } \\
\hline $\begin{array}{l}\text { Laroche et al. } \\
\qquad(2014)^{(103)}\end{array}$ & France & $\begin{array}{l}\text { Early-stage breast cancer and no pain at the start } \\
\text { of Al treatment }\end{array}$ & $\begin{array}{l}\text { Joint pain, diffuse } \\
\text { pain, neuropathic } \\
\text { pain and mixed pain }\end{array}$ & 134 & 77 & 57 & 61 & 7 & $62 \cdot 4$ & $7 \cdot 2$ & 77 & $100 \cdot 0$ & 57 & $100 \cdot 0$ & Hospital-based \\
\hline $\begin{array}{l}\text { Mergenhagen et al. } \\
\quad(2014)^{(104)}\end{array}$ & USA & Patients with statin therapy & Statin-related myalgia & 450 & 50 & 400 & $65 \cdot 5 \ddagger$ & $43-91$ & $68.9 \ddagger$ & $39-96$ & 5 & $10 \cdot 0$ & 16 & $4 \cdot 0$ & Hospital-based \\
\hline $\begin{array}{l}\text { Shantha et al. } \\
(2014)^{(105)}\end{array}$ & USA & Patients with statin therapy & Statin-related myalgia & 1160 & 276 & 884 & 63.5 & $10 \cdot 1$ & $61 \cdot 8$ & $13 \cdot 9$ & 92 & 33.3 & 349 & 39.5 & Community-based \\
\hline Singer et al. (2014) ${ }^{(106)}$ & USA & $\begin{array}{l}\text { Postmenopausal women with non-metastatic, } \\
\text { hormone receptor-positive breast cancer, } \\
\text { prescribed adjuvant Al therapy }\end{array}$ & MSK symptoms & 52 & 28 & 24 & $59 \cdot 8 \ddagger$ & $44-76$ & $61.5 \ddagger$ & $45-76$ & 28 & $100 \cdot 0$ & 24 & $100 \cdot 0$ & Hospital-based \\
\hline Ovesjo et al. $(2016)^{(107)}$ & Sweden & Patients with statin therapy & Myopathy & 127 & 16 & 111 & $65 \S$ & $39-86$ & $65 \S$ & $32-86$ & 12 & $75 \cdot 0$ & 55 & 49.5 & Hospital-based \\
\hline Calza et al. $(2017)^{(108)}$ & Italy & HIV patients with statin therapy & Myalgia & 487 & 42 & 445 & $58 \cdot 6$ & $19 \cdot 6$ & $52 \cdot 5$ & $20 \cdot 2$ & 7 & $16 \cdot 7$ & 83 & $18 \cdot 7$ & Hospital-based \\
\hline
\end{tabular}

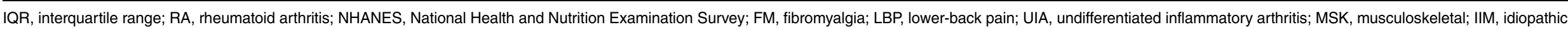
inflammatory myopathies; NHS, Nurses' Health Study; NHSII, Nurses' Health Study II; EIA, early inflammatory arthritis; Al, aromatase inhibitor; CWP, chronic widespread pain; NA, not available.

"One publication reported two studies.

tMedian and IQR.

¥Mean and range.

॥Original article reported age range only. 
Table 2 Association between 25-hydroxyvitamin D concentration and painful conditions

\begin{tabular}{|c|c|c|c|c|c|c|c|c|}
\hline \multirow[b]{2}{*}{ Category } & \multirow[b]{2}{*}{ Subgroup } & \multirow[b]{2}{*}{$\begin{array}{l}\text { No. of included } \\
\text { studies }\end{array}$} & \multicolumn{2}{|c|}{$\begin{array}{c}\text { No. of } \\
\text { participants }\end{array}$} & \multicolumn{2}{|c|}{ Effect estimate } & \multirow[b]{2}{*}{$P$ value } & \multirow{2}{*}{$\begin{array}{l}\text { Test of subgrour } \\
\text { differences, } \\
P \text { value }\end{array}$} \\
\hline & & & $\begin{array}{l}\text { With } \\
\text { pain }\end{array}$ & $\begin{array}{l}\text { Without } \\
\text { pain }\end{array}$ & MD & $95 \% \mathrm{Cl}$ & & \\
\hline \multirow[t]{4}{*}{ Pain conditions } & Arthritis & 33 & 3018 & 3925 & $-12 \cdot 34$ & $-17 \cdot 97,-6 \cdot 71$ & $<0.001$ & \multirow[t]{4}{*}{0.004} \\
\hline & Muscle pain & 22 & 1723 & 3784 & -8.97 & $-14.92,-3.02$ & 0.003 & \\
\hline & Chronic widespread pain & 13 & 4085 & 3306 & $-7 \cdot 77$ & $-11.97,-3.57$ & $<0.001$ & \\
\hline & Headache or migraine & 5 & 4468 & 10063 & -2.53 & $-5.13,0.07$ & 0.06 & \\
\hline \multirow[t]{3}{*}{ Study design } & Cross-sectional & 15 & 7906 & 13457 & $-3 \cdot 10$ & $-4 \cdot 98,-1 \cdot 21$ & 0.001 & \multirow[t]{3}{*}{$<0.001$} \\
\hline & Case-control & 53 & 4976 & 5757 & -11.09 & $-15.25,-6.93$ & $<0.001$ & \\
\hline & Cohort & 5 & 412 & 1864 & -23.55 & $-30 \cdot 68,-16 \cdot 41$ & $<0.001$ & \\
\hline \multirow[t]{2}{*}{ Statin use } & Yes & 11 & 791 & 2733 & $-11 \cdot 15$ & $-20 \cdot 49,-1 \cdot 80$ & 0.02 & \multirow[t]{2}{*}{0.85} \\
\hline & No & 62 & 12503 & 18345 & $-10 \cdot 17$ & $-13 \cdot 17,-7 \cdot 17$ & $<0.001$ & \\
\hline
\end{tabular}

$\mathrm{MD}$, mean difference.

good quality $(33-35,37,39-43,45,48,49,51,54-57,59,60,62,64-70,72-74$, 76-82,84,86-100,102-108), sixteen were satisfactory ${ }^{(31,44,46 \text {, }}$ $47,50,52,53,58,61,63,71,75,83,85,101)$ and the remaining three were unsatisfactory ${ }^{(32,36,38)}$. Specifically, the quality for cross-sectional studies was good for twelve, satisfactory for four and unsatisfactory for three; for case-control studies, nine were very good, thirty-five were good and twelve were satisfactory; and for cohort studies, three were very good and three good. For the unsatisfactory studies, most of them did not have enough information to evaluate the representativeness for the target population. In addition, all the pain-related outcome measurements were based on questionnaire or self-report, and only a few of them validated the pain measurements. The quality assessment scores are shown in Supplement 2, Supplemental Table 1 (see online supplementary material).

\section{Pooled results}

\section{Vitamin D concentration and pain}

For the primary aim, seventy-three studies, containing 13294 participants with pain and 21078 without pain conditions, reported serum $25(\mathrm{OH}) \mathrm{D}$ levels ${ }^{(32,34-40,42}$ $-45,47-50,52-75,77-102,104-108)$. Two publications reported the vitamin $\mathrm{D}$ concentration and pain conditions on different subgroups (smokers and non-smokers ${ }^{(39)}$ or Italian and Estonian ${ }^{(53)}$ ), which are reported separately as four different studies in the current meta-analysis (Supplement 2, Supplemental Table 2).

Compared with controls, mean 25(OH)D concentration was significantly lower in patients with arthritis $(\mathrm{MD}=$ $-12.34 \mathrm{nmol} / 1 ; P<0.001)$, muscle pain $(\mathrm{MD}=-8.97 \mathrm{nmol} / 1$; $P=0.003)$ and chronic widespread pain $(\mathrm{MD}=-7.77 \mathrm{nmol} / \mathrm{l}$; $P<0.001)$, but not in patients with headache or migraine $(\mathrm{MD}=-2.53 \mathrm{nmol} / \mathrm{l} ; P=0.06$; Table 2$)$. These mean differences by disease condition were significantly different $(P=0.004)$. Because of this interaction, forest plots are shown by pain condition rather than for all conditions combined (Fig. 2). Additionally, among age- and sex-matched case-control studies, similar lower vitamin D levels also were observed in patients with arthritis $(\mathrm{MD}=-12.03 \mathrm{nmol} / \mathrm{l}$; $P<0.001)$ and muscle pain (MD $=-11.49 \mathrm{nmol} / 1, P<0.01)$.

In addition, interaction tests were conducted by type of study design and statin use (Table 2; Supplement 2, Supplemental Figs 1 and 2). There was a significant interaction between the three types of study design $(P<0 \cdot 001)$. For each study design, pain patients had significantly lower $25(\mathrm{OH}) \mathrm{D}$ concentration compared with those without pain, but the effect was strongest in cohort studies $(\mathrm{MD}=-23.55 \mathrm{nmol} / 1 ; \quad P<0.001)$, moderate in case-control studies (MD $=-11.09 \mathrm{nmol} / 1 ; P<0.001)$ and weakest in cross-sectional studies $(\mathrm{MD}=-3 \cdot 10 \mathrm{nmol} / \mathrm{l}$; $P=0 \cdot 001$ ). However, there was no interaction between studies of statin users and non-statin users (interaction test $P=0.85)$.

To investigate the impact of other covariables (year, sample size, mean age, female proportion, ratio of participants with and without pain, type of study) on the studylevel estimate of the MD in $25(\mathrm{OH}) \mathrm{D}$ concentration, we performed random-effects meta-regression analyses. We did not observe any significant association for the above covariables and $\mathrm{MD}$ in $25(\mathrm{OH}) \mathrm{D}$ levels in both the univariate and multivariate meta-regression analyses (Supplement 2, Supplemental Table 3).

\section{Vitamin D deficiency and pain}

For the secondary aim, fifty studies reported the proportion of vitamin D deficiency among 14027 patients with pain conditions and 14357 without ${ }^{(31-34,36-38,41-43,46 \text {, }}$ $51,52,54-57,59,61,62,65-69,74-77,79-83,87-89,91-99,103,106,107)$. One publication $^{(76)}$ reported results from two studies which are included separately in the current meta-analysis (Supplement 2, Supplementary Table 4). To maintain consistency with the primary aim analyses (Table 2 ), the secondary aim of vitamin $\mathrm{D}$ deficiency was also analysed by pain condition, study design, statin use and cut-off point for vitamin D deficiency (Table 3 and Fig. 3). The odds of vitamin $\mathrm{D}$ deficiency was increased for arthritis, muscle 


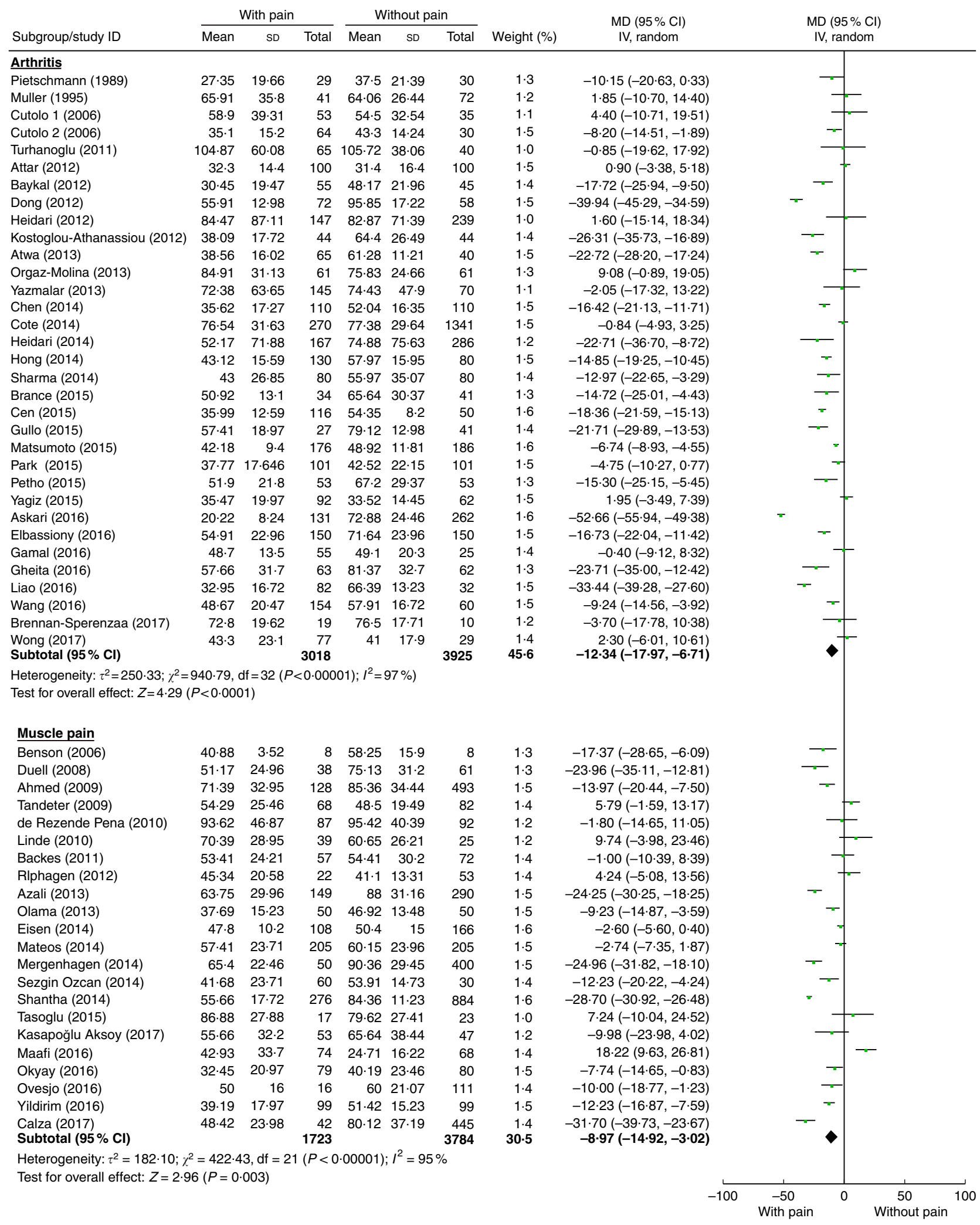

$\mathrm{MD}(95 \% \mathrm{Cl})$

Fig. 2 (colour online) Meta-analysis of the difference in mean serum vitamin D concentration (nmol/l) between participants with and without pain-related conditions. The study-specific mean difference (MD) and $95 \% \mathrm{Cl}$ are represented by the square and horizontal line, respectively; the centre of the diamond represents the pooled $\mathrm{MD}$ and its width represents the pooled $95 \% \mathrm{Cl}$. IV denotes inverse variance; random denotes random-effects model 


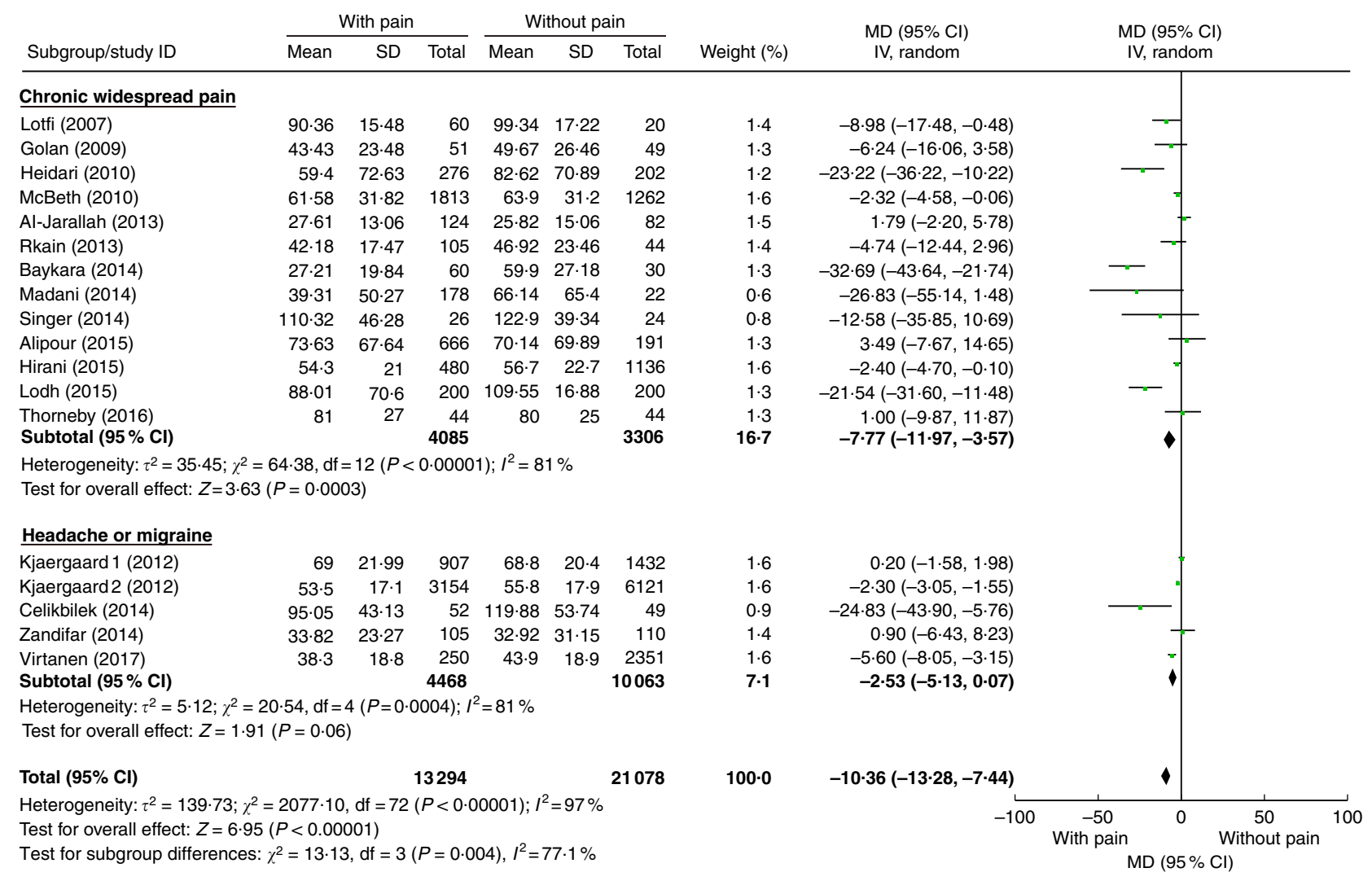

Fig. 2 continued

pain and chronic widespread pain, but not for headache or migraine; and also increased for each of the study designs (cross-sectional, case-control and cohort) and for statin users and non-users separately (Supplement 2, Supplemental Figs 3 and 4). There was a significant interaction associated with the $25(\mathrm{OH}) \mathrm{D}$ cut-off point $(P=0.06)$, with studies that used cut-offs below 50 or $75 \mathrm{nmol} / 1$ reporting significantly increased odds of vitamin D deficiency in patients with pain, but not at a very low cut-off of $<25 \mathrm{nmol} / \mathrm{l}$, although there were only two studies in the latter group (Table 3; Supplement 2, Supplemental Fig. 5). Meta-regression analyses did not find any other covariables that were significantly associated with the $\log (\mathrm{OR})$ of vitamin D deficiency (Supplement 2, Supplemental Table 5).

\section{Sensitivity analysis and publication bias}

For the primary and secondary aims, sensitivity analyses found similar summary measures to those shown in Figs 2 and 3 when studies were individually excluded (see Supplement 2, Supplemental Tables 6 and 7). In addition, after excluding studies with poor quality, we observed similarly lower 25(OH)D levels in arthritis, muscle pain and chronic widespread pain patients than in their controls (see Supplement 2, Supplemental Table 6). There was no convincing evidence of publication bias from funnel plots (Supplement 2, Supplemental Figs 6 and 7), nor from the Egger's test for the primary and secondary aims $(25(\mathrm{OH}) \mathrm{D}$ concentration: $P$ values for publication bias were $0.49,0 \cdot 10,0.17$ and 0.66 for arthritis, muscle pain, chronic widespread pain, and headache or migraine conditions, respectively; while for the proportion of vitamin D deficiency: $P$ values were $0 \cdot 13,0.64$ and 0.06 for arthritis, muscle pain and chronic widespread pain conditions, respectively).

\section{Discussion}

Our results show lower mean $25(\mathrm{OH}) \mathrm{D}$ concentration among patients with widespread chronic pain, muscle pain and arthritis than among their controls (Fig. 2). This result was consistent with the increased odds of hypovitaminosis D associated with these three conditions (Fig. 3). In addition, our study found that the weighted MD in 25(OH)D concentration between patients with pain and control groups is large (arthritis: $12.34 \mathrm{nmol} / \mathrm{l}$ or $20 \%$ difference; muscle pain: $8.97 \mathrm{nmol} / 1$ or $14 \%$ difference; chronic widespread pain: $7.77 \mathrm{nmol} / \mathrm{l}$ or $11.7 \%$ difference) compared with disease-related differences in previous studies, such as those which have reported a $3 \mathrm{nmol} / \mathrm{l}(5 \%)^{(109)}$ and a $7 \mathrm{nmol} / 1$ (11\%) difference ${ }^{(110)}$ between diabetes cases and controls. Overall, these results suggest that low vitamin D status may be associated with the development of painful conditions, with the overall 
Table 3 Association between hypovitaminosis D and painful conditions

\begin{tabular}{|c|c|c|c|c|c|c|c|c|}
\hline \multirow[b]{2}{*}{ Category } & \multirow[b]{2}{*}{ Subgroup } & \multirow[b]{2}{*}{$\begin{array}{l}\text { No. of included } \\
\text { studies }\end{array}$} & \multicolumn{2}{|c|}{$\begin{array}{c}\text { No. of } \\
\text { participants }\end{array}$} & \multicolumn{2}{|c|}{ Effect estimate } & \multirow[b]{2}{*}{$P$ value } & \multirow[b]{2}{*}{$\begin{array}{l}\text { Test of subgroup } \\
\text { differences, } P \text { value }\end{array}$} \\
\hline & & & $\begin{array}{l}\text { With } \\
\text { pain }\end{array}$ & $\begin{array}{c}\text { Without } \\
\text { pain }\end{array}$ & OR & $95 \% \mathrm{Cl}$ & & \\
\hline \multirow[t]{4}{*}{ Pain conditions } & Arthritis & 21 & 2148 & 3411 & $2 \cdot 17$ & $1.56,3.00$ & $<0.001$ & \multirow[t]{4}{*}{0.06} \\
\hline & Muscle pain & 16 & 1052 & 1712 & 2.03 & $1.24,3.33$ & 0.005 & \\
\hline & Chronic widespread pain & 12 & 10722 & 9124 & 1.51 & $1.24,1.85$ & $<0.001$ & \\
\hline & Headache or migraine & 1 & 105 & 110 & 0.89 & $0.45,1.76$ & 0.73 & \\
\hline \multirow[t]{3}{*}{ Study design } & Cross-sectional & 11 & 10500 & 9550 & 1.42 & $1.17,1.73$ & $<0.001$ & \multirow[t]{3}{*}{0.07} \\
\hline & Case-control & 36 & 3406 & 4615 & 2.08 & $1.59,2.73$ & $<0.001$ & \\
\hline & Cohort & 3 & 121 & 192 & $2 \cdot 32$ & $0 \cdot 76,7 \cdot 10$ & 0.14 & \\
\hline \multirow[t]{2}{*}{ Statin use } & Yes & 7 & 659 & 1539 & 1.88 & $1 \cdot 15,3 \cdot 10$ & 0.01 & \multirow[t]{2}{*}{0.94} \\
\hline & No & 44 & 13368 & 12818 & 1.92 & $1.58,2.33$ & $<0.001$ & \\
\hline \multirow[t]{3}{*}{ Cut-off point (nmol/l) } & 25 & 2 & 428 & 639 & 1.26 & $0.93,1.72$ & 0.13 & \multirow[t]{3}{*}{0.06} \\
\hline & 50 & 34 & 9398 & 7573 & 2.08 & $1.59,2.71$ & $<0.001$ & \\
\hline & 75 & 8 & 1999 & 4142 & 1.64 & $1.04,2.57$ & 0.03 & \\
\hline
\end{tabular}

quality of the evidence being rated as moderate according to the Grading of Recommendations Assessment, Development and Evaluation (GRADE) criteria ${ }^{(111)}$ because of the high heterogeneity, although there was no evidence of publication bias, the quality of studies was good and the association was strong (Supplement 2, Supplemental Tables 8 and 9).

Our findings are consistent with previous meta-analyses which found significantly lower 25(OH)D levels in patients on statin therapy with myalgia compared with those without ${ }^{(20)}$ and a positive association between hypovitaminosis D and chronic widespread pain ${ }^{(21)}$. However, by including a further sixty-two studies in our meta-analysis (three studies that were included in the previous meta-analysis were excluded because of controls with pain conditions or no available data), we have extended previous meta-analyses to show that $25(\mathrm{OH}) \mathrm{D}$ levels are also lower in patients with pain not caused by statin therapy - for both the $25(\mathrm{OH}) \mathrm{D}$ concentration and hypovitaminosis D aims (Tables 2 and 3). The evidence for the primary aim from the five cohort studies ${ }^{(104-108)}$, which shows that low vitamin D levels at baseline predicted increased incidence of pain-related conditions (Table 2), supports a possible causal association. In addition, in analyses based on painful conditions, lower mean 25(OH)D levels were found in patients with arthritis, muscle pain and chronic widespread pain (compared with those without pain) for both primary and secondary aims, but not in patients with headache or migraine (Table 2). The latter finding could be due to chance because of the small number of studies (three cross-sectional comparisons from two publications, and two case-control studies) and further research is required to clarify this.

The strengths of the current meta-analysis include: (i) two aims that were predefined at the start of the study; (ii) a search of three electronic databases (MEDLINE, EMBASE and Cochrane Central Register of Controlled Trials), which reduces the possibility of missing relevant articles; (iii) assessment for publication bias by both funnel plots and Egger's test; (iv) inclusion of broader pain conditions, particularly studies of patients with pain who were not on statins; and (v) evaluation of the quality of included studies by the Newcastle-Ottawa scale.

Nevertheless, there are several limitations of the current meta-analysis. Most of the included studies were casecontrol or cross-sectional in design, which could have resulted in reverse causation between pain and lower vitamin D levels. Some studies reported medians, and not means and SD, and information may have been lost in the transfer process. In addition, the included studies lack or have limited adjustment for potential confounders, so the unadjusted association must be interpreted with caution as the spurious associations can be result from potential confounders.

Of major importance is the high heterogeneity observed in the meta-analysis. We tried to identify the sources of this using subgroup, meta-regression and sensitivity analyses, but it remained even when analysing studies by type of pain condition or study design. The high heterogeneity could partly be due to the higher heterogeneity often seen in meta-analyses of observational studies where there is variable control of confounding, compared with randomized controlled trials where effects from standard interventions congregate more closely; and also due to the relatively large number of studies (up to thirty-three) included in the pooled analyses which increases the opportunity for heterogeneity. In our view, this does not lessen the validity of our findings as the results of individual studies almost all go in the same direction (Supplement 2, Supplemental Figs 1 and 2). Further, we used a random-effects model which allows for between-study variation of effect in its calculations.

In addition, the definition of pain varied in each individual publication, so that combining them may also have contributed to the heterogeneity of our results. Therefore, more objective outcome measurements, such as consumption of analgesics as reported in previous studies which have found increased opioid use in people with 


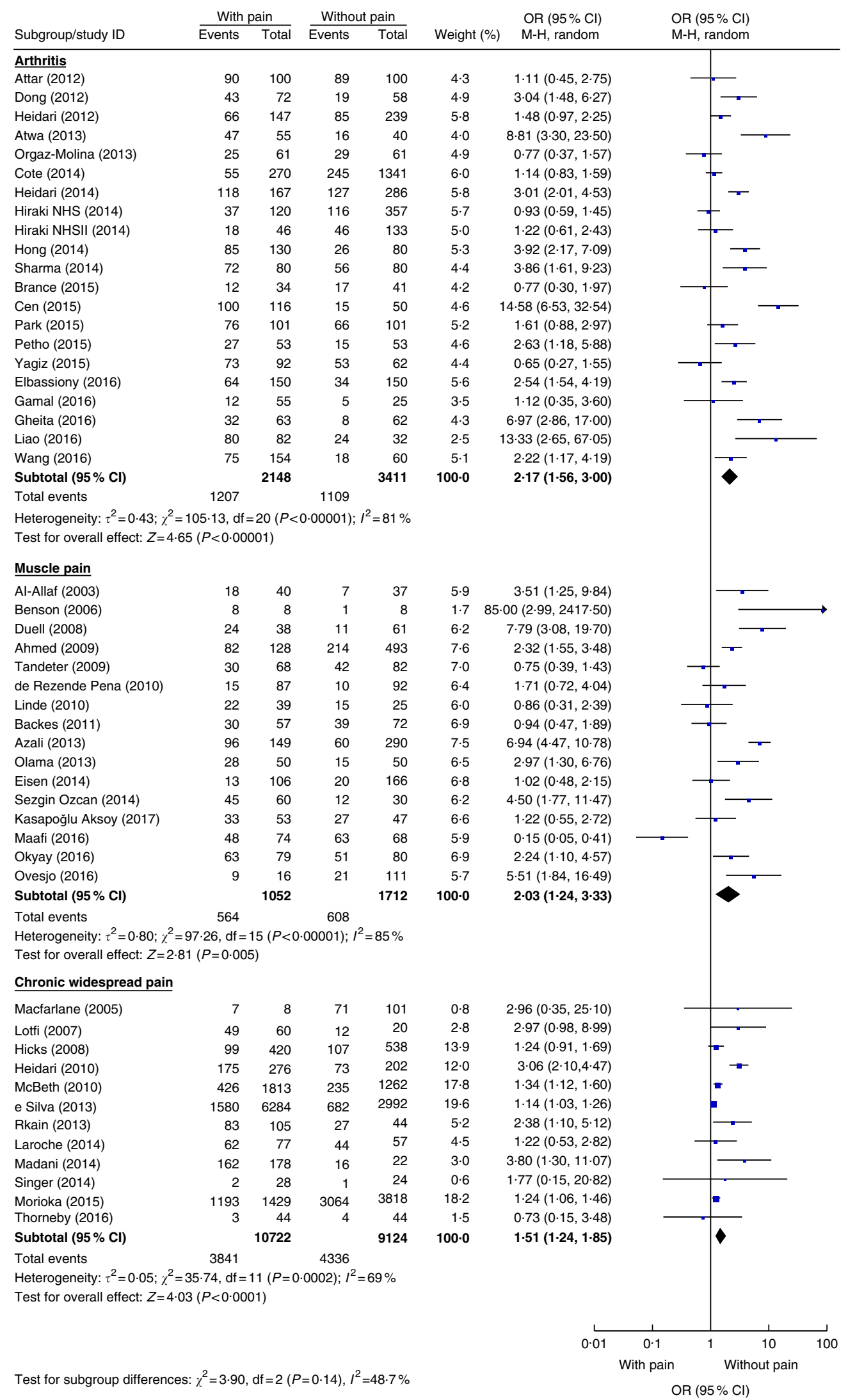

Fig. 3 (colour online) Meta-analysis of the difference in the proportion of vitamin D deficiency between participants with and without pain-related conditions. The study-specific OR and $95 \% \mathrm{Cl}$ are represented by the square and horizontal line, respectively; the centre of the diamond represents the pooled $\mathrm{OR}$ and its width represents the pooled $95 \% \mathrm{Cl}$. M-H denotes Mantel-Haenszel; random denotes random-effects model 
vitamin D deficiency ${ }^{(46,112)}$, or use of validated questionnaires to assess pain severity and function ${ }^{(113)}$, would help to clarify the association between vitamin $\mathrm{D}$ levels and pain in future studies.

\section{Conclusion}

In conclusion, our meta-analysis of eighty-one observational studies suggests that low vitamin D concentration is associated with arthritis, muscle pain and chronic widespread pain. Further well-designed randomized controlled trials should be conducted to confirm the relationship between vitamin D levels and painful conditions.

\section{Acknowledgements}

Financial support: This research received no specific grant from any funding agency in the public, commercial or notfor-profit sectors. Conflict of interest: The authors declare no conflicts of interest related to this study. Authorship: Z.W. conceived the idea, carried out the meta-analyses and drafted the text; Z.M. edited the manuscript and assisted with searching and extracting data; A.W.S. edited the manuscript and advised on statistical methods; C.M.M.L. edited the manuscript; R.S. contributed to the manuscript and provided critical revision to the study. Ethics of buman subject participation: Not applicable.

\section{Supplementary material}

To view supplementary material for this article, please visit https://doi.org/10.1017/S1368980018000551

\section{References}

1. International Association for the Study of Pain (1994) IASP Taxonomy. http://www.iasp-pain.org/Taxonomy\#Pain (accessed January 2017).

2. Ehrlich GE (2003) Low back pain. Bull World Health Organ 81, 671-676.

3. Brooks PM (2006) The burden of musculoskeletal disease a global perspective. Clin Rheumatol 25, 778-781.

4. Stovner L, Hagen K, Jensen R et al. (2007) The global burden of headache: a documentation of headache prevalence and disability worldwide. Cephalalgia 27, 193-210.

5. Phillips CJ (2009) The cost and burden of chronic pain. Rev Pain 3, 2-5.

6. Clauw DJ (2014) Fibromyalgia: a clinical review. JAMA 311, 1547-1555.

7. Hoy D, March L, Brooks P et al. (2014) The global burden of low back pain: estimates from the Global Burden of Disease 2010 study. Ann Rheum Dis 73, 968-974.

8. Cross M, Smith E, Hoy D et al. (2014) The global burden of rheumatoid arthritis: estimates from the global burden of disease 2010 study. Ann Rheum Dis 73, 1316-1322.

9. Borman P \& Celiker R (1999) A comparative analysis of quality of life in rheumatoid arthritis and fibromyalgia. J Musculoskelet Pain 7, 5-14.
10. Arnold LM, Crofford LJ, Mease PJ et al. (2008) Patient perspectives on the impact of fibromyalgia. Patient Educ Couns 73, 114-120.

11. Woolf AD \& Pfleger B (2003) Burden of major musculoskeletal conditions. Bull World Health Organ 81, 646-656.

12. Yelin E (2003) Cost of musculoskeletal diseases: impact of work disability and functional decline. J Rheumatol Suppl 68, $8-11$.

13. Holick MF (2006) High prevalence of vitamin D inadequacy and implications for health. Mayo Clinic Proc 81, 353-373.

14. Simpson RU, Thomas GA \& Arnold AJ (1985) Identification of 1,25-dihydroxyvitamin $\mathrm{D}_{3}$ receptors and activities in muscle. J Biol Chem 260, 8882-8891.

15. Ross AC, Manson JE, Abrams SA et al. (2011) The 2011 report on dietary reference intakes for calcium and vitamin $\mathrm{D}$ from the Institute of Medicine: what clinicians need to know. J Clin Endocrinol Metab 96, 53-58.

16. Hilger J, Friedel A, Herr R et al. (2014) A systematic review of vitamin D status in populations worldwide. Br J Nutr 111, 23-45.

17. Arabi A, El Rassi R \& El-Hajj Fuleihan G (2010) Hypovitaminosis D in developing countries - prevalence, risk factors and outcomes. Nat Rev Endocrinol 6, 550-561.

18. Hossein-nezhad A \& Holick MF (2013) Vitamin D for health: a global perspective. Mayo Clin Proc 88, 720-755.

19. Thacher TD \& Clarke BL (2011) Vitamin D insufficiency. Mayo Clin Proc 86, 50-60.

20. Michalska-Kasiczak M, Sahebkar A, Mikhailidis DP et al. (2015) Analysis of vitamin D levels in patients with and without statin-associated myalgia - a systematic review and meta-analysis of 7 studies with 2420 patients. Int J Cardiol 178, 111-116.

21. Hsiao MY, Hung CY, Chang KV et al. (2015) Is serum hypovitaminosis D associated with chronic widespread pain including fibromyalgia? A meta-analysis of observational studies. Pain Physician 18, E877-E887.

22. Shipton EE \& Shipton EA (2015) Vitamin D deficiency and pain: clinical evidence of low levels of vitamin D and supplementation in chronic pain states. Pain Ther $\mathbf{4}$, $67-87$.

23. Straube S, Derry S, Straube C et al. (2015) Vitamin D for the treatment of chronic painful conditions in adults. Cochrane Database Syst Rev 5, CD007771.

24. Wu Z, Malihi Z, Stewart AW et al. (2016) Effect of vitamin D supplementation on pain: a systematic review and metaanalysis. Pain Physician 19, 415-427.

25. Wells GA, Shea B, O'Connell D et al. (2000) The NewcastleOttawa Scale (NOS) for Assessing the Quality of Nonrandomised Studies in Meta-Analyses. http://www.ohri. $\mathrm{ca} /$ programs/clinical_epidemiology/oxford.asp (accessed January 2017).

26. Takahashi N \& Hashizume M (2014) A systematic review of the influence of occupational organophosphate pesticides exposure on neurological impairment. BMJ Open $\mathbf{4}$, e004798.

27. Wan X, Wang W, Liu J et al. (2014) Estimating the sample mean and standard deviation from the sample size, median, range and/or interquartile range. BMC Med Res Methodol 14, 135.

28. Borenstein M, Hedges LV, Higgins JPT et al. (2010) A basic introduction to fixed-effect and random-effects models for meta-analysis. Res Synth Methods 1, 97-111.

29. Altman DG \& Bland JM (2003) Interaction revisited: the difference between two estimates. BMJ 326, 219.

30. Egger M, Davey Smith G, Schneider M et al. (1997) Bias in meta-analysis detected by a simple, graphical test. BMJ 315, 629-634. 
31. Macfarlane GJ, Palmer B, Roy D et al. (2005) An excess of widespread pain among South Asians: are low levels of vitamin D implicated? Ann Rheum Dis 64, 1217-1219.

32. Duell PB \& Connor WE (2008) Vitamin D deficiency is associated with myalgias in hyperlipidemic subjects taking statins. Circulation 118, S470-S470.

33. Hicks GE, Shardell M, Miller RR et al. (2008) Associations between vitamin $\mathrm{D}$ status and pain in older adults: the Invecchiare in Chianti study. J Am Geriatr Soc 56, 785-791.

34. Ahmed W, Khan N, Glueck CJ et al. (2009) Low serum 25 $(\mathrm{OH})$ vitamin D levels $(<32 \mathrm{ng} / \mathrm{mL})$ are associated with reversible myositis-myalgia in statin-treated patients. Transl Res 153, 11-16.

35. Golan E, Haggiag I, Os P et al. (2009) Calcium, parathyroid hormone, and vitamin D: major determinants of chronic pain in hemodialysis patients. Clin J Am Soc Nephrol 4, 1374-1380.

36. Linde R, Peng L, Desai M et al. (2010) The role of vitamin $\mathrm{D}$ and SLCO1B1*5 gene polymorphism in statin-associated myalgias. Dermatoendocrinology 2, 77-84.

37. McBeth J, Pye SR, O'Neill TW et al. (2010) Musculoskeletal pain is associated with very low levels of vitamin D in men: results from the European Male Ageing Study. Ann Rheum Dis 69, 1448-1452.

38. Backes JM, Barnes BJ, Ruisinger JF et al. (2011) A comparison of 25-hydroxyvitamin D serum levels among those with or without statin-associated myalgias. Atherosclerosis 218, 247-249.

39. Kjaergaard M, Eggen AE, Mathiesen EB et al. (2012) Association between headache and serum 25hydroxyvitamin D: the Tromsø Study: Troms $\varnothing$ 6. Headache 52, 1499-1505.

40. Riphagen IJ, van der Veer E, Muskiet FA et al. (2012) Myopathy during statin therapy in the daily practice of an outpatient cardiology clinic: prevalence, predictors and relation with vitamin D. Curr Med Res Opin 28, $1247-1252$.

41. e Silva AV, Lacativa PG, Russo LA et al. (2013) Association of back pain with hypovitaminosis D in postmenopausal women with low bone mass. BMC Musculoskelet Disord 14, 184 .

42. Eisen A, Lev E, Iakobishvilli Z et al. (2014) Low plasma vitamin $\mathrm{D}$ levels and muscle-related adverse effects in statin users. Isr Med Assoc J 16, 42-45.

43. Madani M, Alavi NM \& Taghizadeh M (2014) Non-specific musculoskeletal pain and vitamin D deficiency in female nurses in Kashan, Iran. I Musculoskelet Pain 22 , 268-274.

44. Alipour M, Hosseini SR, Saadat P et al. (2015) The relationship between chronic musculoskeletal pain and vitamin D deficiency in the elderly population of Amirkola, Iran. J Babol Univ Med Sci 17, 7-14.

45. Hirani V, Blyth FM, Naganathan V et al. (2015) Active vitamin $\mathrm{D}(1,25$ dihydroxyvitamin $\mathrm{D})$ is associated with chronic pain in older Australian men: the Concord Health and Ageing in Men Project. I Gerontol A Biol Sci Med Sci 70, 387-395.

46. Morioka TY, Lee AJ, Bertisch S et al. (2015) Vitamin D status modifies the association between statin use and musculoskeletal pain: a population based study. Atherosclerosis 238, 77-82.

47. Tasoglu O, Kutsal YG, Tasoglu I et al. (2015) Is vitamin D deficiency a risk factor for the development of statininduced myalgia in patients receiving statins? Turk Fiz Tip Rehab D 61, 314-319.

48. Virtanen JK, Giniatullin R, Mantyselka P et al. (2017) Low serum 25 -hydroxyvitamin $\mathrm{D}$ is associated with higher risk of frequent headache in middle-aged and older men. Sci Rep 7, 39697.
49. Pietschmann P, Machold KP, Wolosczuk W et al. (1989) Serum osteocalcin concentrations in patients with rheumatoid arthritis. Ann Rheum Dis 48, 654-657.

50. Muller K, Kriegbaum NJ, Baslund B et al. (1995) Vitamin $\mathrm{D}_{3}$ metabolism in patients with rheumatic diseases: low serum levels of 25-hydroxyvitamin $\mathrm{D}_{3}$ in patients with systemic lupus erythematosus. Clin Rheumatol 14, 397-400.

51. Al-Allaf AW, Mole PA, Paterson CR et al. (2003) Bone health in patients with fibromyalgia. Rheumatology $\mathbf{4 2}$, 1202-1206.

52. Benson J, Wilson A, Stocks N et al. (2006) Muscle pain as an indicator of vitamin D deficiency in an urban Australian Aboriginal population. Med J Aust 185, 76-77.

53. Cutolo M, Otsa K, Laas K et al. (2006) Circannual vitamin d serum levels and disease activity in rheumatoid arthritis: Northern versus Southern Europe. Clin Exp Rheumatol 24, 702-704.

54. Lotfi A, Abdel-Nasser AM, Hamdy A et al. (2007) Hypovitaminosis $\mathrm{D}$ in female patients with chronic low back pain. Clin Rheumatol 26, 1895-1901.

55. Tandeter H, Grynbaum M, Zuili I et al. (2009) Serum 25$\mathrm{OH}$ vitamin D levels in patients with fibromyalgia. Isr Med Assoc J 11, 339-342.

56. de Rezende Pena C, Grillo LP \& das Chagas Medeiros MM (2010) Evaluation of 25-hydroxyvitamin D serum levels in patients with fibromyalgia. Clin Rheumatol 16, 365-369.

57. Heidari B, Shirvani JS, Firouzjahi A et al. (2010) Association between nonspecific skeletal pain and vitamin D deficiency. Int J Rheum Dis 13, 340-346.

58. Turhanoglu AD, Guler H, Yonden Z et al. (2011) The relationship between vitamin $\mathrm{D}$ and disease activity and functional health status in rheumatoid arthritis. Rheumatol Int 31, 911-914.

59. Attar SM (2012) Vitamin D deficiency in rheumatoid arthritis. Prevalence and association with disease activity in Western Saudi Arabia. Saudi Med J 33, 520-525.

60. Baykal T, Senel K, Alp F et al. (2012) Is there an association between serum 25-hydroxyvitamin D concentrations and disease activity in rheumatoid arthritis? Bratisl Lek Listy 113, 610-611.

61. Dong H, Xu L \& Bi L (2012) An investigation on vitamin D levels in peripheral blood in rheumatoid arthritis. Wei Sheng Yen Chiu 41, 313-315.

62. Heidari B, Hajian-Tilaki K \& Heidari P (2012) The status of serum vitamin $\mathrm{D}$ in patients with rheumatoid arthritis and undifferentiated inflammatory arthritis compared with controls. Rheumatol Int 32, 991-995.

63. Kostoglou-Athanassiou I, Athanassiou P, Lyraki A et al. (2012) Vitamin D and rheumatoid arthritis. Ther Adv Endocrinol Metabol 3, 181-187.

64. Al-Jarallah K, Shehab D, Abraham M et al. (2013) Musculoskeletal pain: should physicians test for vitamin D level? Int I Rheum Dis 16, 193-197.

65. Atwa MA, Balata MG, Hussein AM et al. (2013) Serum 25hydroxyvitamin D concentration in patients with psoriasis and rheumatoid arthritis and its association with disease activity and serum tumor necrosis factor- $\alpha$. Saudi Med J 34, 806-813.

66. Azali P, Barbasso Helmers S, Kockum I et al. (2013) Low serum levels of vitamin D in idiopathic inflammatory myopathies. Ann Rheum Dis 72, 512-516.

67. Olama SM, Senna MK, Elarman MM et al. (2013) Serum vitamin D level and bone mineral density in premenopausal Egyptian women with fibromyalgia. Rheumatol Int 33, 185-192.

68. Orgaz-Molina J, Magro-Checa C, Rosales-Alexander JL et al. (2013) Association of 25-hydroxyvitamin D serum levels and metabolic parameters in psoriatic patients 
with and without arthritis. I Am Acad Dermatol 69, 938-946.

69. Rkain H, Bouaddi I, Ibrahimi A et al. (2013) Relationship between vitamin $\mathrm{D}$ deficiency and chronic low back pain in postmenopausal women. Curr Rheumatol Rev 9, 63-67.

70. Yazmalar L, Ediz L, Alpayci M et al. (2013) Seasonal disease activity and serum vitamin D levels in rheumatoid arthritis, ankylosing spondylitis and osteoarthritis. Afr Health Sci 13, 47-55.

71. Baykara B, Dilek B, Nas K et al. (2014) Vitamin D levels and related factors in patients with chronic nonspecific low back pain. J Musculoskelet Pain 22, 160-169.

72. Celikbilek A, Gocmen AY, Zararsiz G et al. (2014) Serum levels of vitamin $D$, vitamin D-binding protein and vitamin $\mathrm{D}$ receptor in migraine patients from central Anatolia region. Int J Clin Prac 68, 1272-1277.

73. Chen J, Liu W, Lin Q et al. (2014) Vitamin D deficiency and low bone mineral density in native Chinese rheumatoid arthritis patients. Int J Rheum Dis 17, 66-70.

74. Cote J, Berger A, Kirchner LH et al. (2014) Low vitamin D level is not associated with increased incidence of rheumatoid arthritis. Rheumatol Int 34, 1475-1479.

75. Heidari B, Heidari P \& Tilaki KH (2014) Relationship between unexplained arthralgia and vitamin $\mathrm{D}$ deficiency: a case control study. Acta Med Iran 52, 400-405.

76. Hiraki LT, Arkema EV, Cui J et al. (2014) Circulating 25hydroxyvitamin D level and risk of developing rheumatoid arthritis. Rheumatology 53, 2243-2248.

77. Hong Q, Xu J, Xu S et al. (2014) Associations between serum 25-hydroxyvitamin D and disease activity, inflammatory cytokines and bone loss in patients with rheumatoid arthritis. Rbeumatology 53, 1994-2001.

78. Mateos F, Valero C, Olmos JM et al. (2014) Bone mass and vitamin $\mathrm{D}$ levels in women with a diagnosis of fibromyalgia. Osteoporos Int 25, 525-533.

79. Sezgin Ozcan D, Oken O, Aras M et al. (2014) Vitamin D levels in women with fibromyalgia and relationship between pain, depression, and sleep. Turk Fiz Tip Rehab $D$ 60, 329-334.

80. Sharma R, Saigal R, Goyal L et al. (2014) Estimation of vitamin D levels in rheumatoid arthritis patients and its correlation with the disease activity. J Assoc Physicians India 62, 678-681.

81. Zandifar A, Masjedi SS, Banihashemi M et al. (2014) Vitamin D status in migraine patients: a case-control study. BioMed Res Int 2014, 514782.

82. Brance ML, Brun LR, Lioi S et al. (2015) Vitamin D levels and bone mass in rheumatoid arthritis. Rheumatol Int $\mathbf{3 5}$, 499-505.

83. Cen X, Liu Y, Yin G et al. (2015) Association between serum 25-hydroxyvitamin D level and rheumatoid arthritis. BioMed Res Int 2015, 913804.

84. Gullo A, Mandraffino G, Bagnato G et al. (2015) Vitamin D status in rheumatoid arthritis: inflammation, arterial stiffness and circulating progenitor cell number. PLoS One 10, e0134602.

85. Lodh M, Goswami B, Mahajan RD et al. (2015) Assessment of vitamin D status in patients of chronic low back pain of unknown etiology. Indian J Clin Biochem 30, 174-179.

86. Matsumoto Y, Sugioka Y, Tada M et al. (2015) Relationships between serum 25 -hydroxycalciferol, vitamin D intake and disease activity in patients with rheumatoid arthritis TOMORROW study. Mod Rheumatol 25, 246-250.

87. Park YE, Kim BH, Lee SG et al. (2015) Vitamin D status of patients with early inflammatory arthritis. Clin Rheumatol 34, 239-246.

88. Petho Z, Kulcsar-Jakab E, Kalina E et al. (2015) Vitamin D status in men with psoriatic arthritis: a case-control study. Osteoporos Int 26, 1965-1970.
89. Yagiz AE, Ustun N, Paksoy H et al. (2015) Association of vitamin $\mathrm{D}$ with disease activity in rheumatoid arthritis and ankylosing spondylitis. J Clin Anal Med 6, 486-489.

90. Askari A, Naghizadeh MM, Homayounfar R et al. (2016) Increased serum levels of IL-17A and IL-23 are associated with decreased vitamin $\mathrm{D}_{3}$ and increased pain in osteoarthritis. PLoS One 11, e0164757.

91. Elbassiony SR, Tawhid Z, Ahmad HS et al. (2016) Serum 25-hydroxy vitamin D levels in Egyptian patients with rheumatoid arthritis: association with disease activity, functional disability and radiological damage. Egyptian Rheumatol 38, 133-139.

92. Gamal RM, Gaber W, Sayed S et al. (2016) Vitamin D status in Egyptian patients with rheumatoid arthritis. Aktuelle Rheumatologie 41, 492-498.

93. Gheita TA, Sayed S, Gheita HA et al. (2016) Vitamin D status in rheumatoid arthritis patients: relation to clinical manifestations, disease activity, quality of life and fibromyalgia syndrome. Int J Rheum Dis 19, 294-299.

94. Kasapoğlu Aksoy M, Altan L \& Okmen Metin B (2017) The relationship between balance and vitamin $25(\mathrm{OH}) \mathrm{D}$ in fibromyalgia patients. Mod Rheumatol 27, 868-874.

95. Liao H, Pan L, Ci W et al. (2016) Clinical significance of serum 25-hydroxyvitamin D in female patients with rheumatoid arthritis. Zhonghua Yi Xue Za Zhi 96, 181-184.

96. Maafi AA, Ghavidel-Parsa B, Haghdoost A et al. (2016) Serum vitamin D status in Iranian fibromyalgia patients: according to the symptom severity and illness invalidation. Korean J Pain 29, 172-178.

97. Okyay R, Kocyigit BF \& Gursoy S (2016) Vitamin D levels in women with fibromyalgia and relationship between pain, tender point count and disease activity. Acta Med Mediterr 32, 243-247.

98. Thorneby A, Nordeman LM \& Johanson EH (2016) No association between level of vitamin $\mathrm{D}$ and chronic low back pain in Swedish primary care: a cross-sectional casecontrol study. Scand J Prim Health Care 34, 196-204.

99. Wang Y, Zhang F, Wang S et al. (2016) Serum vitamin D level is inversely associated with anti-cyclic citrullinated peptide antibody level and disease activity in rheumatoid arthritis patients. Arch Rheumatol 31, 64-70.

100. Yildirim T, Solmaz D, Akgol G et al. (2016) Relationship between mean platelet volume and vitamin D deficiency in fibromyalgia. Biomed Res 27, 1265-1270.

101. Brennan-Speranza TC, Mor D, Mason RS et al. (2017) Skeletal muscle vitamin D in patients with end stage osteoarthritis of the knee. J Steroid Biochem Mol Biol 173, 180-184.

102. Wong TH, Gupta ED, Radhakrishnan AK et al. (2017) Effects of 25-hydroxyvitamin D and vitamin D-binding protein on bone mineral density and disease activity in Malaysian patients with rheumatoid arthritis. Int J Rheum Dis. Published online: 20 February 2017. doi: 10.1111/ 1756-185X.13048.

103. Laroche F, Coste J, Medkour T et al. (2014) Classification of and risk factors for estrogen deprivation pain syndromes related to aromatase inhibitor treatments in women with breast cancer: a prospective multicenter cohort study. J Pain 15, 293-303.

104. Mergenhagen K, Ott M, Heckman K et al. (2014) Low vitamin $\mathrm{D}$ as a risk factor for the development of myalgia in patients taking high-dose simvastatin: a retrospective review. Clin Ther 36, 770-777.

105. Shantha GPS, Ramos J, Thomas-Hemak L et al. (2014) Association of vitamin D and incident statin induced myalgia - a retrospective cohort study. PLoS One $\mathbf{9}$, e88877.

106. Singer O, Cigler T, Moore AB et al. (2014) Hypovitaminosis $\mathrm{D}$ is a predictor of aromatase inhibitor musculoskeletal symptoms. Breast J 20, 174-179. 
107. Ovesjo ML, Skilving I, Bergman P et al. (2016) Low vitamin $\mathrm{D}$ levels and genetic polymorphism in the vitamin D receptor are associated with increased risk of statininduced myopathy. Basic Clin Pharmacol Toxicol 118, 214-218.

108. Calza L, Magistrelli E, Colangeli V et al. (2017) Significant association between statin-associated myalgia and vitamin D deficiency among treated HIV-infected patients. AIDS 31, 681-688.

109. Deleskog A, Hilding A, Brismar K et al. (2012) Low serum 25-hydroxyvitamin D level predicts progression to type 2 diabetes in individuals with prediabetes but not with normal glucose tolerance. Diabetologia 55, 1668-1678.

110. Gagnon C, Lu ZX, Magliano DJ et al. (2011) Serum 25-hydroxyvitamin D, calcium intake, and risk of type 2 diabetes after 5 years: results from a national, population-based prospective study (the Australian
Diabetes, Obesity and Lifestyle study). Diabetes Care 34, $1133-1138$

111. Guyatt GH, Oxman AD, Vist GE et al. (2008) GRADE: an emerging consensus on rating quality of evidence and strength of recommendations. BMJ 336, 924-926.

112. Bergman P, Sperneder S, Hoijer J et al. (2015) Low vitamin $\mathrm{D}$ levels are associated with higher opioid dose in palliative cancer patients - results from an observational study in Sweden. PLoS One 10, e0128223.

113. Hawker GA, Mian S, Kendzerska T et al. (2011) Measures of Adult Pain Visual Analog Scale for Pain (VAS Pain), Numeric Rating Scale for pain (NRS Pain), McGill Pain Questionnaire (MPQ), Short-Form McGill Pain Questionnaire (SF-MPQ), Chronic Pain Grade Scale (CPGS), Short Form-36 Bodily Pain Scale (SF-36 BPS), and Measure of Intermittent and Constant Osteoarthritis Pain (ICOAP). Arthrit Care Res (Hoboken) 63, Suppl. 11, S240-S252. 